\title{
Higher-Order Results for the Relation between Channel Conductance and the Coulomb Blockade for Two Tunnel-Coupled Quantum Dots
}

\author{
John M. Golden and Bertrand I. Halperin \\ Department of Physics, Harvard University, Cambridge, MA 02138
}

(Submitted 15 March 1996)

\begin{abstract}
We extend earlier results on the relation between the dimensionless tunneling channel conductance $g$ and the fractional Coulomb blockade peak splitting $f$ for two electrostatically equivalent dots connected by an arbitrary number $N_{\text {ch }}$ of tunneling channels with bandwidths $W$ much larger than the two-dot differential charging energy $U_{2}$. By calculating $f$ through second order in $g$ in the limit of weak coupling $(g \rightarrow 0)$, we illuminate the difference in behavior of the large- $N_{\mathrm{ch}}$ and small- $N_{\mathrm{ch}}$ regimes and make more plausible extrapolation to the strong-coupling $(g \rightarrow 1)$ limit. For the special case of $N_{\mathrm{ch}}=2$ and strong coupling, we eliminate an apparent ultraviolet divergence and obtain the next leading term of an expansion in $(1-g)$. We show that the results we calculate are independent of such band structure details as the fraction of occupied fermionic single-particle states in the weak-coupling theory and the nature of the cut-off in the bosonized strong-coupling theory. The results agree with calculations for metallic junctions in the $N_{\mathrm{ch}} \rightarrow \infty$ limit and improve the previous good agreement with recent two-channel experiments.
\end{abstract}

PACS: 73.20.Dx,71.45.-d,73.40.Gk

\section{INTRODUCTION}

The opening of tunneling channels between two quantum dots leads to an erosion of the individual dots' Coulomb blockade. 1 For a pair of electrostatically identical quantum dots (see Fig. 1 for a schematic view of the double-dot structure), the progress of this erosion can be chronicled by tracking the splitting of the Coulomb blockade conductance peaks as they evolve from doubly degenerate single-dot conductance resonances to that of nondegenerate double-dot peaks with twice the original periodicity 26 For a system in which the tunneling channels can be treated as having the same individual conductances and in which the Coulomb charging energies are large compared to the single-particle level spacings but small compared to the tunneling channel bandwidths, the fractional peak splitting $f$ can be expressed as a function of two parameters: $N_{\mathrm{ch}}$, the number of tunneling channels between the two dots, and $g$, the dimensionless conductance per tunneling channel. (In this paper, the conductances indicated are always dimensionless conductances, by which we mean the actual conductance divided by the conductance quantum, $e^{2} / h$.)

In particular, for weakly coupled dots $(g \rightarrow 0)$, the fractional peak splitting can be expressed perturbatively as a sum of terms of the form $a_{m, n}\left(N_{\mathrm{ch}}\right)^{m} g^{n}$, where $1 \leq m \leq n$ and $a_{m, n}$ is independent of $N_{\mathrm{ch}}$ and $g$. Previous work 6 has produced the leading term in this expansion. However, as this term is simply linear in the total interdot tunneling conductance, $g_{\text {tot }}=N_{\mathrm{ch}} g$, it does not effectively distinguish between behavior in the large- $N_{\text {ch }}$ and small- $N_{\text {ch }}$ limits. To make such a distinction, one must calculate to second order in $g$, in which case one obtains two sets of terms, one set proportional to $N_{\mathrm{ch}} g^{2}=g_{\text {tot }}^{2} / N_{\mathrm{ch}}$ and the other proportional to
$\left(N_{\mathrm{ch}}\right)^{2} g^{2}=g_{\text {tot }}^{2}$. The $\left(N_{\mathrm{ch}}\right)^{2}$ terms should agree with results from the theory of metallic junctions, in which the leading terms in a large- $N_{\mathrm{ch}}$ expansion are calculated $\mathrm{D}, 8$

In the strong-coupling limit $(g \rightarrow 1)$, a dramatic dependence of the peak splitting on $N_{\text {ch }}$ has already been found. In the cases of $N_{\mathrm{ch}}=1$ and $N_{\mathrm{ch}}=2$, the leading nontrivial terms have been found to be preportional to $\sqrt{1-g}$ and $(1-g) \ln (1-g)$, respectively 6.9 and it has been hypothesized 10,11 that, for $N_{\mathrm{ch}}>2$ but finite, the leading nontrivial term is proportional to $(1-g)^{N_{\mathrm{ch}} / 2}$. This last suggestion appear fo correspond to calculations in the large- $N_{\text {ch }}$ limit, 12.13 where the effective charging energy has been found to be proportional to $e^{-g_{\text {tot }} / 2}$, which is equivalent to $\left(1-g_{\mathrm{tot}} / N_{\mathrm{ch}}\right)^{N_{\mathrm{ch}} / 2}$ in the limit $N_{\text {ch }} \rightarrow \infty$.

Despite the recent progress in study of the strongcoupling limit for the case of most direct experimental interest 2, $10 N_{\mathrm{ch}}=2$, the leading-term calculation fails to be completely satisfactory for at least three reasons. The first is that this calculation does not answer the question of whether the coefficient of $(1-g) \ln (1-g)$ is affected by the manner in which the ultraviolet cut off is imposed in the low-energy Luttinger liquid theory. The second is that the coefficient of the sub-leading term linear in $(1-g)$ is both unknown and naively infinite. Finally, there is the worry - which also applies to the weak-coupling result - that, for $N_{\mathrm{ch}}=2$, interpolation between the solutions for weak- and strong-coupling is difficult because the respective $f$-versus- $g$ curves do not come especially close. 1

This paper addresses these three concerns for the twochannel problem and also extends earlier results for the general $N_{\mathrm{ch}}$-channel problem in the limit of weak coupling. In so doing, it illuminates the difference between large- $N_{\text {ch }}$ and small- $N_{\text {ch }}$ behavior for $g \approx 0$, creates a theory that can be more realistically compared to exper- 
imental results for $N_{\mathrm{ch}}=2$, and argues for the universality of the results, which should be independent of the nature and magnitude of the ultraviolet cut-offs. Section II presents the $g^{2}$ extension of the weak-coupling theory and checks the result against calculations in the $N_{\text {ch }} \rightarrow \infty$ limit. Section III gives the $(1-g)$ correction to the leading dependence in the strong-coupling limit for $N_{\mathrm{ch}}=2$ and shows a plot of the experimental results and revised theoretical predictions for two-channel interdot junctions. Section IV argues that the strong coupling results of Section III are independent of the nature of the way the ultraviolet cut-off is imposed and do not change when one allows the fermionic theory to stray from halffilling. Section V summarizes the results, and Appendices A and B present technical details of calculations in Sections II and III, respectively.

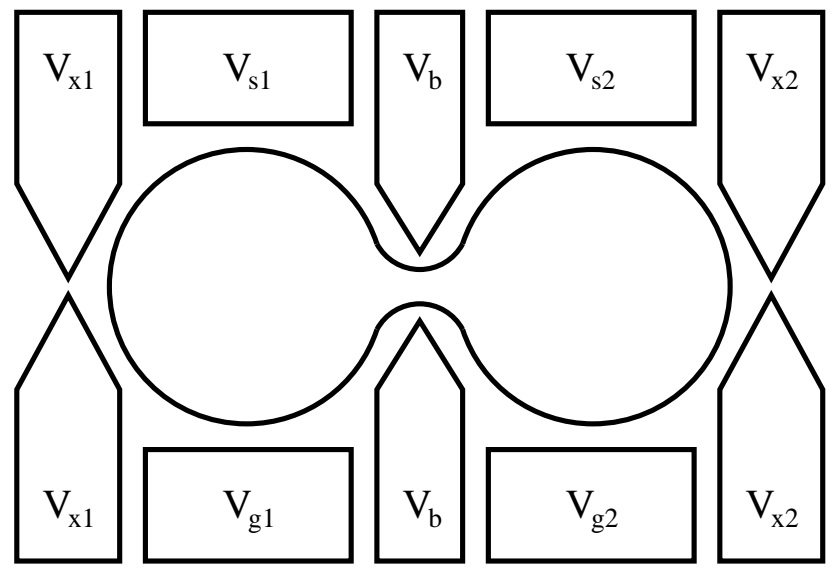

FIG. 1. Schematic diagram for the double dot. Negative potentials are applied to each of the gates to form the double-dot structure. The gate potentials $V_{\mathrm{g} 1}$ and $V_{\mathrm{g} 2}$ control the average numbers of electrons on the dots. These are the potentials that are varied to see the Coulomb blockade. $V_{\mathrm{b}}$ controls the rate of tunneling between the dots. $V_{\mathrm{x} 1}$ and $V_{\mathrm{x} 2}$ control the rate of tunneling to the adjacent bulk two-dimensional electron-gas (2DEG) leads. For calculations of the double-dot energy shifts, tunneling to the leads is assumed negligible compared to tunneling between the two dots. In measuring the total channel conductance $G_{\text {tot }}$, however, the potentials $V_{\text {xi }}$ are turned off so that each dot is strongly connected to its lead. The side-wall potentials $V_{\mathrm{s} 1}$ and $V_{\mathrm{s} 2}$ are fixed.

\section{THE WEAK-COUPLING LIMIT FOR ARBITRARY $N_{\mathrm{CH}}$}

For weakly coupled quantum dots, we use a model "site-to-site" hopping Hamiltoniant and calculate perturbatively in the tunneling term $H_{T}$ :

$$
H=H_{K}+H_{C},
$$

$$
\begin{aligned}
H_{K} & =\sum_{i=1}^{2} \sum_{\sigma} \sum_{\mathbf{k}} \epsilon_{\mathbf{k}} \hat{n}_{i \mathbf{k} \sigma} \\
H_{C} & =U_{2}(\hat{n}-\rho / 2)^{2} \\
H_{T} & =\sum_{\sigma} \sum_{\mathbf{k}_{\mathbf{1}} \mathbf{k}_{\mathbf{2}}} t\left(c_{2 \mathbf{k}_{\mathbf{2}} \sigma}^{\dagger} c_{1 \mathbf{k}_{\mathbf{1}} \sigma}+\text { H.c. }\right) .
\end{aligned}
$$

As in Ref. 4, in these equations, $i$ is the dot index; $\sigma$ is the channel index; $\mathbf{k}$ is the index for all internal degrees of freedom not included in the channel index; $H_{C}$ is the part of the electrostatic potential energy that is affected by interdot tunneling; $\hat{n}$ is half the difference in dot occupation numbers, $\hat{n}=\left(\hat{n}_{2}-\hat{n}_{1}\right) / 2 ; \rho$ is a differential gate voltage parameter and is restricted to values between 0 and 1 (as permitted by the system's unit periodicity); and $U_{2}$ is the differential charging energy, which, for electrostatically equivalent dots, is given by the formula $U_{2}=e^{2} /\left(C_{\Sigma}+2 C_{\text {int }}\right)$, where $C_{\text {int }}$ is the interdot capacitance and $C_{\Sigma}$ is the total single-dot capacitance minus the interdot capacitance. If the dots are not electrostatically equivalent, the formula for $U_{2}$ and the definition of $\rho$ are more complicated. 56 However, the model is still applicable, and the results for $f_{\rho}$ can still be used to obtain the peak splitting.

These calculations are made palatable by assuming that $U_{2}$ is much smaller than the tunneling-channel bandwidth $W$ yet much greater than the average intrachannel level-spacing $\delta$ : $W \gg U_{2} \gg \delta$. This assumption leaves us with a theory that we can consider to be in the continuum limit and that we can hope to be independent of ultraviolet cut-offs. As the bandwidth is presumably of the order of the Fermi energy $\epsilon_{F}$, these assumptions are reaspnable for the micrometer-sized dots of Waugh et al., 2 . 3 for which $\epsilon_{F} \approx 10 \mathrm{meV}, U_{2} \approx 400 \mu \mathrm{eV}$, and $\delta \approx 30 \mu \mathrm{eV}$.

As in Ref. 4, our primary goal is to calculate the fractional peak splitting $f$-i.e., the ratio of the distance between split Coulomb blockade subpeaks for a given $g$ and their maximal separation in the strong-coupling $(g \rightarrow 1)$ limit. It was shown in Ref. 4 that, if the total number of electrons on the two dots is assumed even, the problem of solving for $f$ is a corollary to the problem of solving for a more general quantity $f_{\rho}$, which characterizes the ground-state energy of the double-dot when the difference between the external potentials applied to the dots is nontrivial and the total number of electrons on the two dots is fixed and even. Recall the equation for $f_{\rho}$ :

$$
f_{\rho}=\frac{\Delta_{0}-\Delta_{\rho}}{U_{2} \rho^{2} / 4},
$$

where $\Delta_{\rho}$ is the shift in the ground state energy induced by tunneling at a given value of the gate voltage parameter $\rho$ and $U_{2} \rho^{2} / 4$ is the difference between the unperturbed ground state energies for the given $\rho$ and $\rho=0$. In Ref. 4, it was shown that, for symmetric dots,

$$
f=f_{\rho=1} .
$$


In the same work, it was determined that $f_{\rho}$ exhibits the following leading behavior as $g \rightarrow 0$ :

$$
\begin{gathered}
f_{\rho}^{(1)}=\frac{N_{\mathrm{ch}} g}{\pi^{2} \rho^{2}}[(1-\rho) \ln (1-\rho)+(1+\rho) \ln (1+\rho) \\
\left.+\mathcal{O}\left(\rho^{2} / \psi\right)\right],
\end{gathered}
$$

where $\psi=W / U_{2} \gg 1$. Thus, the corresponding leading behavior for $f$ is

$$
f^{(1)}=\frac{2 \ln 2}{\pi^{2}} N_{\mathrm{ch}} g+\mathcal{O}\left(g / \psi, g^{2}\right) .
$$

Extending perturbation theory beyond this resulti.e., beyond first-order in $g$ - requires some laborious computation. The next-leading contributions come from two sources. The first, which we shall call $f_{\rho}^{(2 A)}$, arises from a combination of the second-order energy shift that has already been calculated and the second term in the formula that relates the tunneling amplitude $t$ to the channel conductance $g$. [The first term in this formula was used to derive Eq. (4).] The second source of $g^{2}$ terms, $f_{\rho}^{(2 B)}$, is the shift in the ground-state energy provided by terms that are fourth-order in $t$.

The first contribution is relatively easy to calculate. The equation for $g$ in terms of $t$ has been derived for half-filling in Ref. 14 and for arbitrary filling in Ref. 4. In the latter calculation, the system is assumed to have a constant density of states between single-particle energies $\epsilon_{0}$ and $\left(\epsilon_{0}+W\right)$, the density of states being zero elsewhere. The system's level of "filling" is then characterized by the filling fraction $F=\left(\epsilon_{F}-\epsilon_{0}\right) / W$, where $\epsilon_{F}$ is the Fermi energy. In accordance with the half-filling result, one then finds the following:

$$
g=\frac{4 \chi}{\left|1+(1+i \eta)^{2} \chi\right|^{2}},
$$

where $\chi=(\pi t / \delta)^{2}$ and $\eta=(1 / \pi) \ln [F /(1-F)]$. Inverting this expression, one discovers that

$$
\frac{t^{2}}{\delta^{2}}=\frac{g}{4 \pi^{2}}\left[1+\frac{1-\eta^{2}}{2} g+\mathcal{O}\left(g^{2}\right)\right] .
$$

Consequently, our first $g^{2}$ term is equal to the right side of Equation (4) multiplied by $\left(1-\eta^{2}\right) g / 2$ :

$$
\begin{aligned}
f_{\rho}^{(2 A)}=\left(1-\eta^{2}\right) & \frac{N_{\mathrm{ch}} g^{2}}{2 \pi^{2} \rho^{2}}[(1-\rho) \ln (1-\rho) \\
& \left.+(1+\rho) \ln (1+\rho)+\mathcal{O}\left(\rho^{2} / \psi\right)\right] .
\end{aligned}
$$

This term is of the expected form $a_{1,2}^{(2 A)} N_{\operatorname{ch}} g^{2}$, where $a_{1,2}^{(2 A)}$ is a function of $\rho$.

On the other hand, $a_{1,2}^{(2 A)}$ is dependent on the filling fraction $F$, a fact which appears to imperil our dreams of a theory that is universal in that it is insensitive to the details of the high-energy behavior (including whether, for example, certain high-energy states exist and therefore have a role in determining the filling fraction $F$ ).
We shall see, however, that the $F$-dependence of $f_{\rho}^{(2 A)}$ actually serves our end, for it exactly cancels the $F$ dependence of $f_{\rho}^{(2 B)}$. As a result, we can further conclude that, through second order in the channel conductance $g$, expression of the fractional peak splitting in terms of the channel conductance is not only convenient for comparison with experiment but is also necessary and sufficient for constructing a result that can be hoped to be universal.

To support this claim, we must actually determine the value of $f_{\rho}^{(2 B)}$. Sadly, it cannot be obtained as effortlessly as $f_{\rho}^{(2 A)}$. There are 24 separate terms that contribute to the fourth-order energy shift. One 12-member subset consists of terms proportional to $\left(N_{\mathrm{ch}}\right)^{2}$; the second consists of those simply linear in $N_{\mathrm{ch}}$. All but four of the twenty-four terms correspond to a specific series of four tunneling events that begin and end with the doubledot system's unperturbed ground state. The remaining four, which belong to the $\left(N_{\mathrm{ch}}\right)^{2}$ subset, correspond to the fourth-order terms in Rayleigh-Schrödinger perturbation theory that are products of the second-order energy shift and a propagator squared. These four have been described by Grabert as diagrams with insertions.

In general, the nature of the twenty-four fourth-order terms is most digestibly summarized via a diagrammatic representation that looks essentially like one of timeordered single-particle diagrams (see Fig. 2). Despite the superficial single-particle nature of this representation, it is important to remember that the propagators that enter into the energy calculations are the propagators for the entire double-dot system, which depend upon both the tunneling particles' individual kinetic energies and the system's multiparticle potential energy. The presence of the multiparticle potential energy makes it impossible to reduce the calculation to the normal Feynman diagrams, for which one can write the problem entirely in terms of single-particle propagators. The presence of exchange terms, which do not appear among the diagrams proportional to $\left(N_{\mathrm{ch}}\right)^{2}$, makes a pseudo-single-particle representation necessary.

Within this time-ordered perturbation theory scheme, the individual fourth-order terms are plagued by both ultraviolet and infrared divergences. Every term is divergent as the bandwidth goes to infinity and four of the $\left(N_{\mathrm{ch}}\right)^{2}$ terms are divergent as $\rho \rightarrow 1$. (A different set of four is divergent as $\rho \rightarrow-1$.) From the result for $f_{\rho}^{(1)}$, we might hope to cancel the ultraviolet divergences and to obtain an answer for the ground-state energy that is infrared-singular but not infrared-divergent. Indeed, as Grabert has noted, the ultraviolet divergences of the $\left(N_{\mathrm{ch}}\right)^{2}$ terms must drop out since, in the limit $U_{2} \rightarrow 0$, these terms correspond to disconnected diagrams or insertion diagrams that exactly cancel one another and thus do not appear as Feynman diagrams. In contrast, the $N_{\text {ch }}$ diagrams do have nontrivial Feynman-diagram analogs. As a whole, they correspond to a single totemic Feynman diagram - an individual ring marked by four 


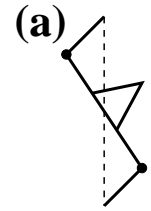

(1)

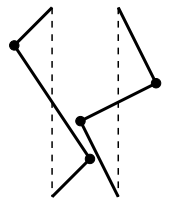

(4)

(b)

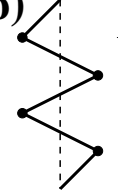

(1)

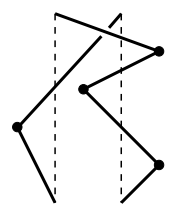

(4)

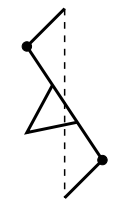

(2)

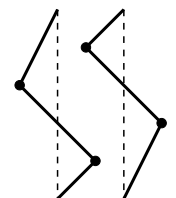

(5)

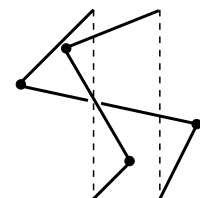

(2)

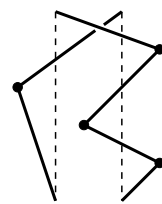

(5)

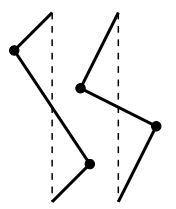

(3)

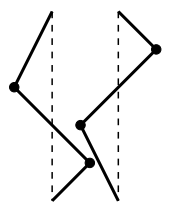

(6)

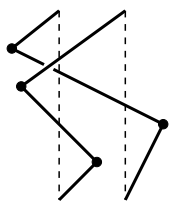

(3)

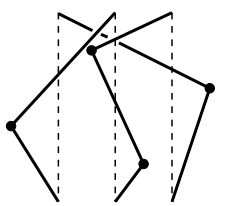

(6)
FIG. 2. Diagrams for half of the (a) fourth-order, $\left(N_{\mathrm{ch}}\right)^{2}$ terms and (b) fourth-order, $N_{\mathrm{ch}}$ terms. The remaining terms are represented by diagrams that are mirror images of these. A vertical dashed line is drawn for each of the $m$ particles that tunnels at least once from one dot to the other. This line stands for the corresponding particle's initial state, a state that must be filled at the end of the four tunneling events in order to recover the unperturbed ground state from which the system starts. A particle begins at the bottom of its vertical initial-state line. Particles in dot 1 propagate upward and rightward. Particles in dot 2 propagate upward and leftward. A tunneling event for a particle is signaled by a solid dot that coincides with a bend in the particle-propagation path. Each particle must end on one of the dashed vertical lines, meaning that it ends in the single-particle state that corresponds to that line. Insertions (see Sec. II) are represented by triangles that project off a single-particle propagation line. If the projection points up, the insertion corresponds to the term in the second-order energy shift for which a particle tunnels off the dot occupied by the propagating particle. If the projection points down, the insertion corresponds to the second-order term for which a particle tunnels onto the dot occupied by the propagating particle. In the absence of exchange, all particles end on their own initial-state lines. A two-particle exchange carries a minus sign and results in each of two particles ending on the other's initial-state line. Three-particle exchange carries no sign (alternatively, one can view it as carrying two canceling minus signs) and results in each of three particles ending on one of the others' initial-state lines. tunneling events. The ultraviolet divergences of these diagrams are therefore expected to be persistent but irrelevant because we are interested only in the relative shift between the ground-state energies for arbitrary $\rho$ and for $\rho=0$ (recall Eq. 2). Accordingly, we expect that, when one subtracts the fourth-order shift for $\rho=0$ from that for arbitrary $\rho$, the fourth-order terms produce a result that is neither ultraviolet- nor infrared-divergent but is infrared-singular as $|\rho| \rightarrow 1$. A brief summary of the actual calculation of these terms follows. Those interested in more detail are invited to peruse Appendix A, which offers a fuller description of the calculation of the $\left(N_{\mathrm{ch}}\right)^{2}$ diagrams and a step-by-step computation of the contribution from one representative $N_{\mathrm{ch}}$ term.

For the less scrupulous, there are still a few facts worthy of note. A prominent feature of the fourth-order calculation is that each term involves the integration over four energy variables $\left(\epsilon_{i}\right.$, where $i$ ranges from 1 to 4$)$ of a product of three propagators. In the $\left(N_{\mathrm{ch}}\right)^{2}$ diagrams, the energy variables "pair off": $\epsilon_{1}$ and $\epsilon_{3}$ only appear as parts of the combination $\epsilon_{\mathrm{I}}=\left(\epsilon_{3}-\epsilon_{1}\right)$, and $\epsilon_{2}$ and $\epsilon_{4}$ only appear as parts of the combination $\epsilon_{\mathrm{II}}=\left(\epsilon_{4}-\epsilon_{2}\right)$. As a result, calculation of these terms reduces to the performance of double integrations over $\epsilon_{\mathrm{I}}$ and $\epsilon_{\mathrm{II}}-$ albeit with a nontrivial density of states.

The $N_{\text {ch }}$ diagrams cannot be handled in this way, for they involve particle exchanges that frustrate any desire to pair off the energy variables. The quadruple integration over the $\epsilon_{i}$ cannot be eluded. It can, however, be expedited by differentiating twice with respect to $\rho$ while integrating out the energy variables and, then, integrating twice with respect to $\rho$ in the end. One might worry about the fact that, by differentiating twice with respect to $\rho$, one has lost knowledge of terms constant and linear in $\rho$. However, these terms are unimportant. As noted in Ref. 4, the ground-state energy (perturbed or unperturbed) is symmetric in $\rho$. Therefore, terms linear in $\rho$ must cancel out of the fourth-order energy shift when all the terms are summed. Constant terms are similarly negligible since, as usual, we are only interested in the relative energy shift $\Delta_{\rho}-\Delta_{0}$.

After the aforementioned tricks for calculating the $\left(N_{\mathrm{ch}}\right)^{2}$ and $N_{\mathrm{ch}}$ diagrams have been used, the only real wrinkles that remain are integrals of the form

$$
\mathcal{P} \int_{0}^{R \psi} d x \frac{\ln (x+B)}{(x+A)},
$$

where $0 \leq|A|<B, R$ is either $F$ or $(1-F)$, and, as before, $\psi=W / U_{2}$. The symbol $\mathcal{P}$ indicates that, for $A<0$, only the principal value of the integral is calculated. These integrals can be done by rewriting the argument of $\ln (x+B)$ as $[(x+A)+(B-A)]$ and Taylorexpanding about $(x+A)$ for $(B-A)<|x+A|$ and about $(B-A)$ for $(B-A)>|x+A|$. The result of such an integration may be sensitive to whether the system is below half-filling $[F<(1-F)]$, at half-filling $[F=(1-F)]$, or above half-filling $[F>(1-F)]$. However, the system 
as a whole has particle-hole symmetry, so one expects that the final result - once all the terms are summed - is symmetric under exchange of $F$ and $(1-F)$. If there is no jump discontinuity when the system is precisely half full, the result for $F<(1-F)$ should determine the answer for all "finite" $F$, by which we mean all $F$ such that $F \psi,(1-F) \psi \gg 1$. This thesis has been explicitly confirmed.

Indeed, the $\left(N_{\mathrm{ch}}\right)^{2}$ part of the fourth-order relative energy shift is found to be independent of the filling fraction. Its contribution to $f_{\rho}$ has a rather lengthy explicit form:

$$
\begin{aligned}
f_{\rho,\left(N_{\mathrm{ch}}\right)^{2}}^{(2 B)}= & \frac{\left(N_{\mathrm{ch}}\right)^{2} g^{2}}{4 \pi^{4} \rho^{2}}\left\{-\frac{\pi^{2}}{6} \rho^{2}+4(1-\rho) \ln (1-\rho)\right. \\
& +\frac{1-\rho^{2}}{2} \ln ^{2}(1-\rho)-2(2-\rho) \ln [2(2-\rho)] \\
& +\frac{1+\rho^{2}}{2} \ln (1+\rho) \ln (1-\rho) \\
& -2 \ln (3-\rho) \ln (1-\rho)+\ln ^{2}(3-\rho) \\
& +\frac{(3-\rho)(1-\rho)}{2}[\ln (1-\rho)-\ln (3-\rho)]^{2} \\
& -\left(5-4 \rho+\rho^{2}\right) \sum_{n=1}^{\infty} \frac{(-1)^{n+1}}{n^{2}}\left(\frac{1-\rho}{3-\rho}\right)^{n} \\
& -\frac{5 \ln ^{2} 3}{2}+8 \ln 2+5 \kappa \\
& +[\rho \rightarrow-\rho]\}
\end{aligned}
$$

where the contents of the last pair of brackets indicate that one sums over all the terms in the curly braces again after replacing $\rho$ with $-\rho$ and the quantity $\kappa$ is given by

$$
\kappa=\sum_{n=1}^{\infty} \frac{(-1)^{n+1}}{n^{2}}\left(\frac{1}{3}\right)^{n} .
$$

It has been confirmed numerically that the derivative of $f_{\rho,\left(N_{\mathrm{ch}}\right)^{2}}^{(2 B)}$ agrees with a calculation by Grabert in the $N_{\text {ch }} \rightarrow \infty$ limit 8 More precisely, the derivative of this term with respect to $\rho$ corresponds exactly to Grabert's calculation of the leading corrections to the ground-state expectation value of $\hat{n}$. The value at $\rho=0$ of the second derivative of this term with respect to $\rho$ (and of the first derivative with respect to $\rho$ of Grabert's $\hat{n}$ correction) should agree with the large- $N_{\text {ch }}$ "effective capacitance" of Golubev and Zaikin. 4 However, this is found not to be so, the $g^{2}$ correction calculated by Golubev and Zaikin being nearly a factor of 20 smaller than that computed here. The present calculation therefore provides an important check on the large- $N_{\text {ch }}$ calculations, resolving an apparent contradiction in the literature.

There are no corroborating calculations for the terms that are linear in $N_{\mathrm{ch}}$ as these are negligible in the large$N_{\text {ch }}$ limit. However, knowing that $f=1$ when $g=1$ and that $f^{(1)} \approx(0.14) N_{\mathrm{ch}} g$, one might conjecture that the sign of the $g^{2}$ term changes from positive to negative when $N_{\text {ch }}$ is of order 10 . With respect to the expansion of $f$, such a crossover would imply that the coefficient of $N_{\mathrm{ch}} g^{2}$ is positive and approximately 10 times the size of the negative coefficient of $\left(N_{\mathrm{ch}}\right)^{2} g^{2}$.

To check this conjecture, we need to know the value of the fourth-order, linear-in- $N_{\mathrm{ch}}$ contribution to the fractional peak splitting. Our results for this quantity are

$$
\begin{aligned}
f_{\rho, N_{\mathrm{ch}}}^{(2 B)}= & \frac{N_{\mathrm{ch}} g^{2}}{4 \pi^{4} \rho^{2}}\left\{2 \pi^{2} \eta^{2}(1-\rho) \ln (1-\rho)+\frac{4}{3}(1-\rho) \ln ^{3}(1-\rho)\right. \\
& -2(2+\ln 2)(1-\rho) \ln ^{2}(1-\rho) \\
& +4\left(2+\ln 2-\ln 2 \ln 3-\kappa+\frac{\pi^{2}}{3}\right)(1-\rho) \ln (1-\rho) \\
& -2\left(\frac{\pi^{2}}{3}-2 \kappa-2 \ln 2 \ln 3\right)[(3-\rho) \ln (3-\rho)-3 \ln 3] \\
& -\frac{2}{3}\left[(3-\rho) \ln ^{3}(3-\rho)-3(3-\rho) \ln ^{2}(3-\rho)\right. \\
& \left.\quad+6(3-\rho) \ln (3-\rho)-3 \ln ^{3} 3+9 \ln { }^{2} 3-18 \ln 3\right] \\
& -2 \ln 2[(3-\rho) \ln 2(3-\rho)-2(3-\rho) \ln (3-\rho) \\
& \left.\quad-3 \ln ^{2} 3+6 \ln 3\right] \\
& -2 \sum_{i=1}^{11} A_{i}(\rho) \\
& +[\rho \rightarrow-\rho]\}
\end{aligned}
$$

where $\kappa$ is given by Eq. (10), $\eta=(1 / \pi) \ln [F /(1-F)]$, and the $A_{i}(\rho)$ are defined below:

$$
\begin{aligned}
& A_{1}(\rho)=-\int_{0}^{\rho} d x \frac{(\rho-x) \ln ^{2}(3-x)}{1-x} \\
& A_{2}(\rho)=2 \int_{0}^{\rho} d x \frac{(\rho-x) \ln (3-x) \ln (1-x)}{1-x} \\
& A_{3}(\rho)=-2 \ln 2 \int_{0}^{\rho} d x \frac{(\rho-x) \ln (3-x)}{1-x} \\
& A_{4}(\rho)=2(1-\rho) \int_{0}^{\rho} d x \ln \left(\frac{2-x}{3-x}\right) \ln \left(\frac{1-\rho}{1-x}\right) \\
& \times\left(\frac{1}{3-x}-\frac{1}{1-x}\right) \\
& A_{5}(\rho)=2 \int_{0}^{\rho} d x(\rho-x) \ln \left(\frac{2-x}{3-x}\right)\left(\frac{1}{3-x}-\frac{1}{1-x}\right) \\
& A_{6}(\rho)=\int_{0}^{\rho} d x \frac{(\rho-x) \ln ^{2}(1-x)}{3-x} \\
& A_{7}(\rho)=-2 \int_{0}^{\rho} d x \frac{(\rho-x) \ln (3-x) \ln (1-x)}{3-x} \\
& A_{8}(\rho)=-2 \ln 2 \int_{0}^{\rho} d x \frac{(\rho-x) \ln (1-x)}{3-x} \\
& A_{9}(\rho)=-2(3-\rho) \int_{0}^{\rho} d x \ln \left(\frac{2-x}{3-x}\right) \ln \left(\frac{3-\rho}{3-x}\right) \\
& \times\left(\frac{1}{3-x}-\frac{1}{1-x}\right) \\
& A_{10}(\rho)=-2 \int_{0}^{\rho} d x(\rho-x) \ln \left(\frac{2-x}{3-x}\right)
\end{aligned}
$$




$$
\begin{array}{r}
\times\left(\frac{1}{3-x}-\frac{1}{1-x}\right) \\
A_{11}(\rho)=-\int_{0}^{\rho} d x \ln ^{2}(1-x) \ln (1+x)
\end{array}
$$

The characterization of the fourth-order energy shift is now essentially complete. The result is more unwieldy than we would like. However, there are a few highlights that are easy to draw out. As expected, the fourth-order shift is neither ultraviolet- nor infrared-divergent but is singular as $|\rho| \rightarrow 1$, the leading singularities being in agreement, with an earlier calculation by Glazman and Matveev. 19 In addition and quite gratifyingly, the solution is independent of the filling fraction $F$. As discussed earlier, the dependence of $f_{\rho}^{(2 B)}$ on the filling fraction, which is concentrated in the $\eta^{2}$ term of the first line of Eq. (11), exactly cancels that of Eq. (8). Hence, there is some reason to believe that, when expressed in terms of the channel conductance $g$, the result is universal in the sense that it is independent of the details of the band structure for energies much greater than $U_{2}$, where $U_{2}$ is much less than the bandwidth $W$.

It is difficult to get a better handle on this algebraic smorgasbord by mere inspection. One can add some precision to the picture of what has been accomplished by first assembling the $g^{2}$ terms of $f_{\rho}$ and then plugging in $\rho=1$ to obtain the contribution to the symmetric-dot fractional peak splitting $f$. Upon recalling that

$$
f_{\rho}^{(2)}=f_{\rho}^{(2 A)}+f_{\rho,\left(N_{\mathrm{ch}}\right)^{2}}^{(2 B)}+f_{\rho, N_{\mathrm{ch}}}^{(2 B)},
$$

one can evaluate the $A_{\mathrm{i}}$ integrals numerically for $\rho=1$ to obtain

$$
f^{(2)} \approx[0.1491] N_{\mathrm{ch}} g^{2}-[0.009798]\left(N_{\mathrm{ch}}\right)^{2} g^{2} .
$$

We see that the conjecture about the $\left(N_{\mathrm{ch}}\right)^{2}$ and $N_{\mathrm{ch}}$ contributions to $f^{(2)}$ is correct: the terms have opposite sign, and the ratio of their magnitudes is on the order of 10. For the case of $N_{\mathrm{ch}}=2$, the $g^{2}$ term provides the desired upward correction to the $f$-versus- $g$ curve.

Before specializing to the result for $N_{\mathrm{ch}}=2$, we should explore the consequences of having a term proportional to $N_{\mathrm{ch}} g^{2}$. This term makes the result sensitive to the "fine structure" of the interdot conductance. As remarked in the introduction, terms of the form $\left(N_{\mathrm{ch}} g\right)^{n}$ can be rewritten as a simple power of the total conductance between the dots: $\left(N_{\mathrm{ch}} g\right)^{n}=\left(g_{\mathrm{tot}}\right)^{n}$. Should the conductances in the various tunneling channels be allowed to differ, the form of these terms when written in terms of $g_{\text {tot }}$ would remain unchanged. The only alteration would be in the equation for $g_{\text {tot }}$ itself, which would revert to the more fundamental form

$$
g_{\mathrm{tot}}=\sum_{\sigma} g_{\sigma}
$$

where $g_{\sigma}$ denotes the dimensionless conductance of the $\sigma$ th channel.
For terms proportional to $\left(N_{\mathrm{ch}}\right)^{m} g^{n}$ with $m \neq n$, the situation is quite different. Consider the $N_{\mathrm{ch}} g^{2}$ term in Eq. (13). If we had suspended the sum over channels until the end of our calculation, we would have seen that these terms are proportional to

$$
\left[g^{2}\right]_{\mathrm{tot}}=\sum_{\sigma} g_{\sigma}^{2}
$$

Only when symmetry considerations constrain all the individual channel conductances to be equal can we safely use $\left[g^{2}\right]_{\mathrm{tot}}=\left(g_{\mathrm{tot}}\right)^{2} / N_{\mathrm{ch}}$.

Consequently, for the general situation in which the conductances in the separate channels are not necessarily equal, the appropriate equation for the fractional peak splitting is the following:

$$
f \approx 0.1405\left(g_{\mathrm{tot}}\right)+0.1491\left[g^{2}\right]_{\mathrm{tot}}-0.009798\left(g_{\mathrm{tot}}\right)^{2}+\ldots
$$

If we extended the expression to $n$th order in the dimensionless conductances, it would contain factors such as

$$
\left[g^{m}\right]_{\mathrm{tot}}=\sum_{\sigma} g_{\sigma}^{m}
$$

where $m \leq n$ and these factors appear both alone and in combination up to $n$th order in dimensionless conductance. The details of the "fine structure" are fully characterized by the set of $\left[g^{m}\right]_{\text {tot }}$ for $1 \leq m \leq N_{\mathrm{ch}}$, and the fractional peak splitting can be expressed in terms of these. Further modifications might be thought necessary to account for the "hyperfine structure" that results from allowing the tunneling amplitude $t$ in Eq. (11) to be a nontrivial function of $\mathbf{k}_{\mathbf{1}}$ and $\mathbf{k}_{\mathbf{2}}$. However, as long as the tunneling amplitude varies little over an energy range of order $U_{2}$, one would not expect Eq. (16) to be changed substantially.

\section{THE STRONG-COUPLING LIMIT FOR $N_{\mathrm{CH}}=2$}

The $g^{2}$ correction to the two-channel solution boosts confidence in the small- $g$ end of our $f$-versus- $g$ interpolation (see Fig. 3) but does little to improve the precision of theoretical predictions in the strong-coupling limit, a fact of particular concern for the experimentally relevant case of two interdot tunneling channels.2. 10 The sections of the paper that follow improve the strong-coupling theory for $N_{\mathrm{ch}}=2$ in two substantial ways. The first contribution, presented here in Sec. III, is the calculation of the second term in the $(1-g)$ expansion about the $g=1$ ground state. This term, which is linear in $(1-g)$, is of interest both because it is significant in determining the shape of the $f$-versus- $g$ curve and because, in the cal culation that yields the primary $(1-g) \ln (1-g)$ term, 
the $(1-g)$ term is naively ultraviolet-divergent. The second important contribution, which comes in Sec. IV, is the provision of powerful evidence that the coefficients of the leading terms in the $(1-g)$ expansion are indeed independent of the high-energy structure of the theory.

To calculate in the limit of $g \rightarrow 1$, we model the tunneling link between the dots as a one-dimensional channel with a delta function scattering potential at its center. This model was originally developed for the problem of a single dot connected to a bulk lead 11 but was shown in Refs. 4 and 5 to be easily adaptable to that of a pair of coupled dots. Within this ansatz, the value of the double-dot charging energy is a simple reflection of the total number of electrons that have been transferred through this channel from one side of the barrier $(\operatorname{dot} 1)$ to the other (dot 2). In addition, as the system is effectively one-dimensional, the fermionic degrees of freedom can be bosonized, and the Euclidean action assumes a characteristic Luttinger-liquid form 11 , 15 .

$$
\begin{aligned}
S & =S_{0}+S_{\mathrm{int}}+S_{\mathrm{b}} \\
S_{0} & =\frac{1}{\beta} \sum_{\sigma} \sum_{\omega_{m}}\left|\omega_{m}\right|\left|\tilde{\theta}_{\sigma}\left(\omega_{m}\right)\right|^{2} \\
S_{\mathrm{int}} & =U_{2} \int_{0}^{\beta} d \tau\left(\frac{1}{\sqrt{\pi}}\left[\sum_{\sigma} \theta_{\sigma}(\tau)\right]-\frac{\rho}{2}\right)^{2}, \\
S_{\mathrm{b}} & =\frac{\tilde{V} W}{2 \pi} \sum_{\sigma} \int_{0}^{\beta} d \tau \cos \left[2 \sqrt{\pi} \theta_{\sigma}(\tau)\right] .
\end{aligned}
$$

In these formulas, $\theta_{\sigma}(\tau)$ is a bosonic field that tracks the displacement of the one-dimensional electron gas at the barrier $(x=0)$, and $\tilde{\theta}_{\sigma}\left(\omega_{m}\right)$ is its Fourier transform:

$$
\theta_{\sigma}(\tau)=\frac{1}{\beta} \sum_{\omega_{m}} e^{-i \omega_{m} \tau} \tilde{\theta}_{\sigma}\left(\omega_{m}\right),
$$

where $\tau$ is an imaginary time divided by $\hbar, \beta$ is the inverse temperature $\left(\beta=1 / \mathrm{k}_{\mathrm{B}} T\right)$, and $\omega_{m}$ is $\hbar$ times a bosonic Matsubara frequency $\left(\omega_{m}=2 \pi m / \beta\right)$. In addition, $\tilde{V}$ is a measure of the barrier strength defined by $\tilde{V}=\frac{V_{0}}{\hbar v_{F}}$ for the delta-function potential $V_{0} \delta(x)$. As for the remaining parameters, $v_{F}$ is the Fermi velocity and, as in the weakcoupling theory, $W$ is the bandwidth - the difference between the lowest and highest single-particle energies in the channel. The inverse temperature $\beta$ will be taken to infinity in calculating the energy of the ground state.

As in the weak-coupling theory, we ultimately want to parametrize the coupling between the dots by the dimensionless channel conductance $g$, rather than the barrier strength $\tilde{V}$. Accordingly, we need to find the relation between $g$ and $\tilde{V}$. In our single-mode channel, $g$ equals the single-particle transmission probability $T$, and $(1-g)$ equals the reflection probability $R$. The leading dependence of the channel conductance on $\tilde{V}$ equals what one would guess from the reflection probability of a single particle incident upon a one-dimensional delta-function potential16:

$$
(1-g)=\tilde{V}^{2}+\mathcal{O}\left(\tilde{V}^{4}\right)
$$

Inverting this formula, we find that

$$
\tilde{V}^{2}=(1-g)+\mathcal{O}\left[(1-g)^{2}\right]
$$

To lowest order, we have the approximation of Matveev, 9 $\tilde{V}=\sqrt{1-g}$, which-it will be seen-is all that is required for the calculations in this paper.

Having prepared ourselves to switch from a solution in terms of $\tilde{V}$ to one in terms of $g$, we proceed with the calculation of the ground-state energy. Our first move is to reorganize the action, expressing it in terms of bosonic fields that characterize the net charge and pseudospin degrees of freedom, where the pseudospin degrees of freedom correspond to "true spin" only if the two channels correspond to spin-up and spin-down, respectively. Defining the charge field by $\theta_{\mathrm{c}}=\theta_{1}+\theta_{2}+\sqrt{\pi} \rho / 2$ and the pseudospin field by $\theta_{\mathrm{s}}=\theta_{1}-\theta_{2}$, we find

$$
\begin{aligned}
S & =S_{0}^{(\mathrm{s})}+S_{0}^{(\mathrm{c})}+S_{\mathrm{b}}, \\
S_{0}^{(\mathrm{s})} & =\frac{1}{2 \beta} \sum_{\omega_{m}}\left|\omega_{m}\right|\left|\tilde{\theta}_{\mathrm{s}}\left(\omega_{m}\right)\right|^{2}, \\
S_{0}^{(\mathrm{c})}= & \frac{1}{2 \beta} \sum_{\omega_{m}}\left(\left|\omega_{m}\right|+\frac{2 U_{2}}{\pi}\right)\left|\tilde{\theta}_{\mathrm{c}}\left(\omega_{m}\right)\right|^{2}, \\
S_{\mathrm{b}}= & \frac{\tilde{V} W}{\pi} \int_{0}^{\beta} d \tau \cos \left[\sqrt{\pi} \theta_{\mathrm{c}}(\tau)+\frac{\pi \rho}{2}\right] \\
& \quad \times \cos \left[\sqrt{\pi} \theta_{\mathrm{s}}(\tau)\right] .
\end{aligned}
$$

The Euclidean action has now been written in terms of "high-energy" charge modes and "low-energy" pseudospin modes. We advance by integrating out the "highenergy" charge degrees of freedom. This integration is analogous to a renormalization in which one integrates out the higher-energy degrees of freedom within a particular channel 17 One begins with the generating functional for the Euclidean action of Eq. (21):

$$
Z=\int D\left[\theta_{\mathrm{s}}(\tau)\right] \int D\left[\theta_{\mathrm{c}}(\tau)\right] e^{-S\left[\theta_{\mathrm{s}}(\tau), \theta_{\mathrm{c}}(\tau)\right]},
$$

where, as usual, time-ordering is implicit within the functional integral approach. One then performs the integration over the fast modes to obtain the generating functional for an effective action depending only on the slow modes:

$$
\begin{aligned}
Z_{\mathrm{s}} & =\int D\left[\theta_{\mathrm{s}}(\tau)\right] e^{-S_{\text {eff }}\left[\theta_{\mathrm{s}}(\tau)\right]} \\
e^{-S_{\mathrm{eff}}\left[\theta_{\mathrm{s}}(\tau)\right]} & =\frac{e^{-S_{0}^{(\mathrm{s})}} \int D\left[\theta_{\mathrm{c}}(\tau)\right] e^{-S_{0}^{(\mathrm{c})}} e^{-S_{\mathrm{b}}}}{\int D\left[\theta_{\mathrm{c}}(\tau)\right] e^{-S_{0}^{(\mathrm{c})}}} .
\end{aligned}
$$

Eq. 23) determines the effective action $S_{\text {eff. }}$. To solve for it, one Taylor-expands the exponential factor $e^{-S_{\mathrm{b}}}$, performs the integral over charge degrees of freedom, and 
re-exponentiates the result. Before doing any of this, it is useful to make the following definition:

$$
\langle\hat{A}\rangle_{\mathrm{c}}=\frac{\int D\left[\theta_{\mathrm{c}}(\tau)\right] \hat{A} e^{-S_{0}^{(\mathrm{c})}}}{\int D\left[\theta_{\mathrm{c}}(\tau)\right] e^{-S_{0}^{(\mathrm{c})}}} .
$$

One can then rewrite Eq. (23) as follows:

$$
\begin{aligned}
e^{-S_{\mathrm{eff}}} & =e^{-S_{0}^{(\mathrm{s})}}\left\langle e^{-S_{\mathrm{b}}}\right\rangle_{\mathrm{c}} \\
& =e^{-S_{0}^{(\mathrm{s})}}\left[1-\left\langle S_{\mathrm{b}}\right\rangle_{\mathrm{c}}+\frac{1}{2}\left\langle S_{\mathrm{b}}^{2}\right\rangle_{\mathrm{c}}+\mathcal{O}\left(\tilde{V}^{3}\right)\right] .
\end{aligned}
$$

Upon re-exponentiation, one obtains

$$
S_{\mathrm{eff}}=S_{0}^{(\mathrm{s})}+\left\langle S_{\mathrm{b}}\right\rangle_{\mathrm{c}}-\frac{1}{2}\left\langle\left[S_{\mathrm{b}}-\left\langle S_{\mathrm{b}}\right\rangle_{\mathrm{c}}\right]^{2}\right\rangle_{\mathrm{c}}+\mathcal{O}\left(\tilde{V}^{3}\right) .
$$

It is clear that to solve for the effective action to order $\tilde{V}^{2}=(1-g)$, we must solve for both corrections to $S_{0}^{(\mathrm{s})}$ on the right side of Eq. (26).

Details of the calculation of these terms are presented in Appendix B. The result is that

$$
S_{\mathrm{eff}}=S_{0}^{(\mathrm{s})}+S_{\mathrm{b}}^{(1)}+S_{\mathrm{b}}^{(2)},
$$

where

$$
\begin{gathered}
S_{\mathrm{b}}^{(1)}=\frac{\tilde{V} W}{\pi} e^{-\frac{\pi}{2} K_{\mathrm{c}}(0)} \cos \left(\frac{\pi \rho}{2}\right) \int_{0}^{\beta} d \tau \cos \left[\sqrt{\pi} \theta_{\mathrm{s}}(\tau)\right] \\
S_{\mathrm{b}}^{(2)}=\left(\frac{\tilde{V} W}{\pi}\right)^{2} e^{-\pi K_{\mathrm{c}}(0)} \int_{0}^{\beta} d \tau_{1} \int_{0}^{\tau_{1}} d \tau_{2} \\
\times\left\{\cos ^{2}\left(\frac{\pi \rho}{2}\right)\left[1-e^{-\pi K_{\mathrm{c}}\left(\tau_{1}-\tau_{2}\right)}\right]\right. \\
\left.\quad-\sinh \left[\pi K_{\mathrm{c}}\left(\tau_{1}-\tau_{2}\right)\right]\right\} \\
\times \cos \left[\sqrt{\pi} \theta_{\mathrm{s}}\left(\tau_{1}\right)\right] \cos \left[\sqrt{\pi} \theta_{\mathrm{s}}\left(\tau_{2}\right)\right] .
\end{gathered}
$$

The function $K_{\mathrm{c}}(\tau)$ that appears in these formulas is the charge-channel correlation function, $K_{\mathrm{c}}(\tau)=$ $\left\langle\theta_{\mathrm{c}}(\tau) \theta_{\mathrm{c}}(0)\right\rangle_{\mathrm{c}}$. Its numerical value can be found from the formula

$$
K_{\mathrm{c}}(\tau)=\frac{1}{\pi} \operatorname{Re} \int_{0}^{\infty} d \omega \frac{e^{-\left(\frac{2}{W}+i \tau\right) \omega}}{\omega+\frac{2 U_{2}}{\pi}} .
$$

To progress further, we define a new "unperturbed action" $S_{\text {New }}=S_{0}^{(\mathrm{s})}+S_{\mathrm{b}}^{(1)}$. We then write down the Hamiltonian that corresponds to this action:

$$
\begin{aligned}
H_{\text {New }} & =H_{0}^{(\mathrm{s})}+H_{\mathrm{b}}^{(1)}, \\
H_{\mathrm{b}}^{(1)} & =\frac{\tilde{V} W}{\pi} e^{-\frac{\pi}{2} K_{\mathrm{c}}(0)} \cos \left(\frac{\pi \rho}{2}\right) \cos \left[\sqrt{\pi} \theta_{\mathrm{s}}(0)\right] .
\end{aligned}
$$

This is the Hamiltonian diagonalized by Matveev in Ref. 9 through a process of "debosonization" (see Appendix B) in which the Hamiltonian is rewritten in terms of fermion operators $d_{k}$ and $d$ :

$$
\begin{aligned}
& H_{0}^{(\mathrm{s})}=\int_{-\Lambda}^{\Lambda} d k \xi_{k} d_{k}^{\dagger} d_{k} \\
& H_{\mathrm{b}}^{(1)}=\lambda \int_{-\Lambda}^{\Lambda} d k\left[d_{k}^{\dagger}\left(d+d^{\dagger}\right)+\left(d+d^{\dagger}\right) d_{k}\right] .
\end{aligned}
$$

Here the single-particle energy $\xi_{k}$, the fermion interaction parameter $\lambda$, and the wave-vector cut-off $\Lambda$ have the formulas $\xi_{k}=\hbar v_{F} k, \lambda=\tilde{V} \cos (\pi \rho / 2) \sqrt{2 e^{\gamma} \hbar v_{F} U_{2} / \pi^{3}}$, and $\Lambda=W / 2 \hbar v_{F}$.

Since the Hamiltonian is now quadratic in fermion operators, a Bogoliubov transformation brings it to the desired diagonal form:

$$
H_{\mathrm{New}}=E_{\mathrm{New}}^{(0)}+\int_{0}^{\Lambda} d k \xi_{k}\left(C_{k}^{\dagger} C_{k}+\tilde{C}_{k}^{\dagger} \tilde{C}_{k}\right)
$$

where, if we write down only the terms of lowest order in $\tilde{V}$, replacing all others by an ellipsis,

$$
\begin{aligned}
\tilde{C}_{k} & =\frac{1}{\sqrt{2}}\left(d_{k}+d_{-k}^{\dagger}\right), \\
C_{k} & =\frac{1}{\sqrt{2}}\left(d_{k}-d_{-k}^{\dagger}\right)+\ldots
\end{aligned}
$$

The correction to the $\tilde{V}=0$ ground-state energy is produced by the omitted terms in $C_{k}$ (for details, see Appendix B). In particular, using $\Delta_{\text {str }}^{(1)}$ to represent the difference between the ground-state energies of $H_{\text {New }}$ for $\tilde{V}=0$ and for arbitrary $\tilde{V}$, respectively, one finds that

$$
\begin{aligned}
\Delta_{\text {str }}^{(1)}(\rho)=\frac{4 e^{\gamma} U_{2}}{\pi^{3}} \tilde{V}^{2} \cos ^{2}\left(\frac{\pi \rho}{2}\right) & \\
\times & \left(\ln \left[\tilde{V}^{2} \cos ^{2}\left(\frac{\pi \rho}{2}\right)\right]-\ln \left[\frac{\psi}{2}\right]\right. \\
& \left.-1+\ln \left[\frac{8 e^{\gamma}}{\pi^{2}}\right]\right) .
\end{aligned}
$$

As before, $\psi=W / U_{2}$, where the bandwidth $W \gg U_{2}$.

We see that the result for this first correction contains terms that are quadratic in $\tilde{V}$ and logarithmically divergent in $\psi$. This ultraviolet divergence is circumvented in Ref. 9 by the statement that one should replace $W$ by $U_{2}$ because keeping only the first term from chargechannel integration is only a good approximation for energies less than the charging energy $U_{2}$. The terms in Eq. (33) that are merely quadratic in $\tilde{V}$ are thereby rendered finite and can be dropped in favor of the leading $\tilde{V}^{2} \cos ^{2}(\pi \rho / 2) \ln \left[\tilde{V}^{2} \cos ^{2}(\pi \rho / 2)\right]$ dependence.

To eliminate the logarithmic divergence more formally, one must calculate the shift in the ground-state energy that is induced by $S_{\mathrm{b}}^{(2)}$ [recall Eq. (27)]. As this term is itself quadratic in $\tilde{V}$ and as we are only interested in knowing the ground-state energy to order $\tilde{V}^{2}$, we can drop all but the leading part of the $S_{\mathrm{b}}^{(2)}$-induced shift. In expressing $S_{\mathrm{b}}^{(2)}$ in terms of the diagonalizing operators of $H_{\mathrm{New}}$, one may use the truncated formulas of 
Eq. 322. The relevant shift in the ground-state energy is then found by calculating the expectation value of $S_{\mathrm{b}}^{(2)}$ in the ground state of $H_{\mathrm{New}}$ (see Appendix B):

$$
\begin{aligned}
\Delta_{\mathrm{str}}^{(2)}(\rho)= & \frac{4 e^{\gamma} U_{2}}{\pi^{3}} \tilde{V}^{2} \cos ^{2}\left(\frac{\pi \rho}{2}\right) \\
& \times \int_{0}^{\infty} d x\left[1-e^{-\pi K_{\mathrm{c}}\left(\frac{2 x}{W}\right)}\right] \frac{1-e^{-x}}{x},
\end{aligned}
$$

where units have been chosen such that $\hbar=1$ and terms independent of $\rho$ have been dropped since they are not relevant to evaluation of the fractional peak splitting $f$. It is not too hard to see that the factor $\left[1-e^{-\pi K_{\mathrm{c}}(2 x / W)}\right]$ in the integrand makes for an ultraviolet cut-off of order $\psi=W / U_{2}$ (see Appendix B). It is even easier to see that $\left(1-e^{-x}\right)$ provides an infrared cut-off of order 1. Thus, one can surmise that the leading term from the integral is $\ln (\psi / 2)$, which is precisely what is needed to cancel the ultraviolet divergence in $\Delta_{\mathrm{str}}^{(1)}$.

What remains is to calculate the rest of the integral in Eq. 34, which we call $\Phi$ :

$$
\begin{aligned}
\Phi=\lim _{\psi \rightarrow \infty}\left(\int_{0}^{\infty}\right. & d x\left[1-e^{-\pi K_{c}\left(\frac{2 x}{W}\right)} \frac{1-e^{-x}}{x}\right. \\
- & \left.\ln \left[\frac{\psi}{2}\right]\right) .
\end{aligned}
$$

Numerical approximation of the integral in the limit $\psi \rightarrow \infty$ gives $\Phi=0.1703 \pm 0.0002$.

One can now sum $\Delta_{\text {str }}^{(1)}$ and $\Delta_{\text {str }}^{(2)}$ to get the strongcoupling energy shift through order $\tilde{V}^{2}$. Having dropped terms that are independent of $\rho$, one has

$$
\begin{gathered}
\Delta_{\text {str }}(\rho)=\frac{4 e^{\gamma} U_{2}}{\pi^{3}} \tilde{V}^{2} \cos ^{2}\left(\frac{\pi \rho}{2}\right)\left(\ln \left[\tilde{V}^{2} \cos ^{2}\left(\frac{\pi \rho}{2}\right)\right]\right. \\
\left.-1+\Phi+\ln \left[\frac{8 e^{\gamma}}{\pi^{2}}\right]\right)+\ldots
\end{gathered}
$$

We can now straightforwardly compute the fractional peak-splitting $f$ in terms of the dimensionless conductance $g$. As mentioned before, if we are only interested in obtaining the ground-state energy to order $(1-g)$, only the leading term of Eq. (20) is relevant in converting Eq. (36) to an expression in terms of $(1-g)$. The value of $f=f_{\rho=1}$ follows from the fact that, in the strongcoupling limit,

$$
f_{\rho}=1-\frac{\Delta_{\mathrm{str}}(\rho)-\Delta_{\mathrm{str}}(0)}{U_{2} \rho^{2} / 4} .
$$

In particular, Eqs. (20), (36), and (37) yield

$$
\begin{aligned}
f= & 1+\frac{16 e^{\gamma}}{\pi^{3}}(1-g) \ln (1-g) \\
& -\frac{16 e^{\gamma}}{\pi^{3}}\left[1-\ln \left(\frac{8 e^{\gamma}}{\pi^{2}}\right)-\Phi\right](1-g)+\ldots
\end{aligned}
$$

Since $\Phi \approx 0.1703$, we have $f \approx 1+0.919(1-g) \ln (1-g)-0.425(1-g)+\ldots$

Having determined the first corrections to the leading behaviors for both $g \rightarrow 0$ and $g \rightarrow 1$, we now have a more plausible picture for the connection between the $N_{\mathrm{ch}}=2$ weak- and strong-coupling limits (see Fig. 3). The fit to the data could be improved if the interdot fapacitance were larger than experimentally estimated 3 or if asymmetry between the dots were important. 10 In any case, whether or not such further emendations should be made, the theory is within the range of present experimental error. The corrections introduced in this paper have moved the weak- and strong-coupling predictions by reasonable amounts in the desired directions, increasing both the ease and the precision of interpolation between the weak- and strong-coupling limits.

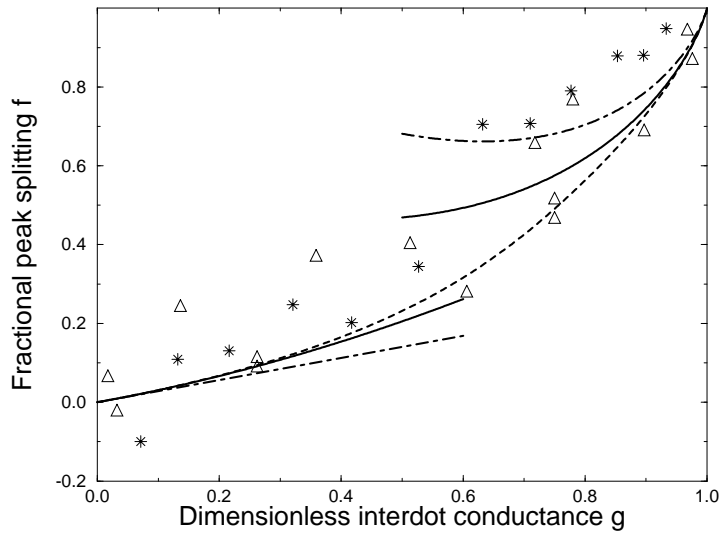

FIG. 3. Graph of the fractional Coulomb blockade conductance peak splitting $f$ as a function of the dimensionless conductance per channel $g$ in the weak- and strong-tunneling limits for $N_{\mathrm{ch}}=2$. The new theoretical curves are depicted as solid lines. The old theoretical curves from Refs. 4 and 5 are dot-dashed lines. The dashed curve shows a possible interpolating function. Data points from Refs. 2 and 3 are given as triangles or stars; the two different symbols correspond to different data sets. The value of $f$ for the experimental data has been extracted from the measured splitting fraction $f^{\prime}$ by using the method discussed in Ref. 4 with experimentally estimated values of $20 \mathrm{aF}$ for the constant interdot capacitance and $0.4 \mathrm{fF}$ for the total single-dot capacitance. ${ }^{2,3}$

\section{INSENSITIVITY TO THE HIGH-ENERGY DENSITY OF STATES}

\section{A. Insensitivity to Functional Form of Bosonic Cut-Off}

To have confidence that our coupled-dot calculations can be usefully compared to empirical data, we should 
make sure that the result, $f$ expressed as a function of $g$, is independent of the details of the band structure far from the Fermi surface, where the assumption of a constant density of states becomes invalid. We have done much to confirm such robustness in the regime of weak coupling, for we have shown there that, through second order in $g, f(g)$ is independent of the bandwidth $W$ and the filling fraction $F$ as long as both $F W$ and $(1-F) W$ are much larger than the charging energy $U_{2}$. Such dual invariance indicates that we can simply shear off a nontrivial number of high-energy states without affecting the result. We would expect then that we could make less Draconian modifications of the high-energy density of states with similarly perfect impunity.

With regard to the strong-coupling theory, matters have been left less assured. In Ref. 4, we introduced a factor of $C_{2}$ multiplying the first term in Eq. (38) to guard against the possibility that the coefficient of the energy shift calculated in the Luttinger-liquid approach was partly a product of the approach itself and, in particular, the manner in which the ultraviolet cut-off was imposed. Concern about such a possibility arises from the fact that the leading term in the $(1-g)$ expansion is proportional to the product of $e^{-\frac{\pi}{2} K_{\mathrm{c}}(0)}$ and $\mathcal{A}$, where $\mathcal{A}$ is the generalization to non-exponential cut-offs of the normalization factor in Eq. (B10) that gives the proportionality between the fermionic position operators and the exponentials of bosonic fields:

$$
\psi_{f}^{\dagger}(0, \tau)=\mathcal{A} e^{i \sqrt{\pi} \phi_{f}(\tau)} .
$$

Changing the nature of the bosonic cut-off [e.g., from the exponential $e^{-\alpha|\omega|}$ to the Gaussian $\left.e^{-(\pi / 4) \alpha^{2} \omega^{2}}\right]$ causes the value of $e^{-\frac{\pi}{2} K_{\mathrm{c}}(0)}$ to be multiplied by a constant factor. Although one would hope that a similar shift in the value of $\mathcal{A}$ compensates for the change in $e^{-\frac{\pi}{2} K_{c}(0)}$, to the authors knowledge, such a happy circumstance has not previously been checked to be true.

Similar questions could be asked about the prefactor for the term linear in $(1-g)$, with which we associate a factor $C_{3}$, where $C_{3}=1$ for the Luttinger-liquid approach with the standard exponential cut-off. This term is proportional both to $|\mathcal{A}|^{2} e^{-\pi K_{\mathrm{c}}(0)}$ and to an integral that depends upon $e^{-\pi K_{\mathrm{c}}(\tau)}$ [see Eq. (34) in Sec. III and Eq. (B26) in Appendix B]. Hence, in order to prove that the two leading strong-coupling terms do not vary with the choice of cut-off function, one must show that neither the product $\mathcal{D}_{1}=|\mathcal{A}|^{2} e^{-\pi K_{\mathrm{c}}(0)}$ nor the integral

$$
\begin{array}{rl}
\mathcal{D}_{2}=\frac{1}{\beta W} \int_{0}^{\beta W / 2} & d x\left(\frac{\beta W}{2}-x\right) \\
& \times\left[1-e^{-\pi K_{\mathrm{c}}(2 x / W)}\right] \frac{1-e^{-x}}{x}
\end{array}
$$

assumes different values when the shape of the cut-off is changed. Though we do not have a general proof that $\mathcal{D}_{1}$ and $\mathcal{D}_{2}$ are independent of the cut-off function, we can show that they remain the same for a whole class of functions that includes the exponential cut-off and that they are similarly unchanged when one replaces the exponential cut-off by a Gaussian. We believe that these facts are convincing evidence that the prefactors in Eq. (38) are insensitive to the nature of the high-energy cut-off.

First, we prove that $\mathcal{D}_{1}$ and $\mathcal{D}_{2}$ are the same for all cut-offs of the form

$$
\nu\left(\omega, \alpha,\left\{b_{m}\right\}\right)=e^{-\alpha|\omega|}\left(1+\sum_{m=1}^{M} b_{m} \alpha^{m}|\omega|^{m}\right),
$$

where either $M$ is finite or, for large $m, b_{m}$ falls to zero faster than $m^{-\zeta} / m$ ! for some real $\zeta>0$. As usual, it is assumed that $\alpha U_{2} \ll 1$, where $\alpha=2 / W$. We add the further assumption that $\left[(m-1) ! b_{m} \alpha U_{2}\right] \ll 1$ for all $m$.

The first step in our proof is to solve for the change in $e^{-\pi K_{\mathrm{c}}(0)}$ when one goes from the standard exponential cut-off $\nu(\omega, \alpha,\{0\})$ to the more general form $\nu\left(\omega, \alpha,\left\{b_{m}\right\}\right)$. The formula for $K_{\mathrm{c}}(\tau)$ [recall Eq. 228)] becomes

$$
K_{\mathrm{c}}(\tau)=\frac{1}{\pi} \operatorname{Re} \int_{0}^{\infty} d \omega \nu\left(\omega, \alpha,\left\{b_{m}\right\}\right) \frac{e^{-i \tau \omega}}{\omega+\frac{2 U_{2}}{\pi}} .
$$

We can write the change in $K_{\mathrm{c}}(0)$ as

$$
\delta K_{\mathrm{c}}(0)=\frac{1}{\pi} \sum_{m=1}^{M} b_{m} \int_{0}^{\infty} d \omega \frac{\alpha^{m} \omega^{m} e^{-\alpha \omega}}{\omega+\frac{2 U_{2}}{\pi}} .
$$

Using $\omega=\left(\omega+2 U_{2} / \pi-2 U_{2} / \pi\right)$ and the binomial theorem, we can expand $\omega^{m}$ in powers of $\left(\omega+2 U_{2} / \pi\right)$. The integration is then straightforward and yields

$$
\pi \delta K_{\mathrm{c}}(0)=\sum_{m=1}^{M} b_{m}(m-1) !\left[1+\mathcal{O}\left(\alpha U_{2}\right)\right] .
$$

Dropping the correction, we have the result

$$
e^{-\pi K_{\mathrm{c}}(0)}=\left[e^{-\sum b_{m}(m-1) !}\right] e^{-\pi K_{\mathrm{c}, 0}(0)},
$$

where $K_{\mathrm{c}, 0}(\tau)$ is the correlation function for the standard exponential cut-off.

Calculation of the change in the normalization com stant $\mathcal{A}$ is more complicated. Following V. J. Emery,22 we find

$$
|\mathcal{A}|^{-2}=\int_{-\infty}^{\infty} d x e^{\int_{0}^{\infty} d \omega \nu\left(\omega, \alpha,\left\{b_{m}\right\}\right) \frac{e^{i \omega x / \hbar v_{F}-1}}{\omega}}+\text { c.c. }
$$

A bit of calculation reveals that

$$
\begin{array}{r}
|\mathcal{A}|^{-2}=\left[e^{-\sum_{m=1}^{M} b_{m}(m-1) !}\right] \int_{-\infty}^{\infty} d x\left(\frac{\alpha}{\alpha-i x}\right) \\
\times e^{\sum b_{m}(m-1) !\left(\frac{\alpha}{\alpha-i x}\right)^{m}}+\text { c.c. }
\end{array}
$$

It is apparent that the bracketed factor exactly cancels the factor that multiplies $e^{-\pi K_{\mathrm{c}, 0}(0)}$ in Eq. (46). Thus, 
in order for $\mathcal{D}_{1}=|\mathcal{A}|^{2} e^{-\pi K_{\mathrm{c}}(0)}$ to be unaltered, the value of the integral in Eq. (48) cannot change as the $b_{m}$ are varied. In short, the partial derivative of the integral with respect to each of these coefficients must be zero. The partial derivative with respect to $b_{m}$ is given by the following formula:

$$
\begin{aligned}
P_{m}=(m-1) ! & \int_{-\infty}^{\infty} d x\left(\frac{\alpha}{\alpha-i x}\right)^{m+1} \\
& \times e^{\sum b_{m}(m-1) !\left(\frac{\alpha}{\alpha-i x}\right)^{m}}+\text { c.c. }
\end{aligned}
$$

Let $z=\alpha /(\alpha-i x)$. The resulting integral in the complex $z$ plane follows a closed path, beginning and ending at $z=0$ :

$$
P_{m}=-i \alpha(m-1) ! \oint d z z^{m-1} e^{\sum b_{m}(m-1) ! z^{m}}+c . c .
$$

For $M$ finite or $b_{m}$ falling off faster than $m^{-\zeta} / m$ !, the integrand is analytic throughout the region enclosed by the contour. By Cauchy's Theorem, $P_{m}=0$.

Since the integral of Eq. (48) does not vary with $b_{m}$, we can make the statement

$$
|\mathcal{A}|^{2}=\left[e^{\sum_{m=1}^{M} b_{m}(m-1) !}\right]\left|\mathcal{A}_{0}\right|^{2},
$$

where $\mathcal{A}_{0}$ is the normalization factor for the standard exponential cut-off. Eqs. (46) and (51) yield

$$
\mathcal{D}_{1}=|\mathcal{A}|^{2} e^{-\pi K_{\mathrm{c}}(0)}=\left|\mathcal{A}_{0}\right|^{2} e^{-\pi K_{\mathrm{c}, 0}(0)} .
$$

We have now shown that, for the class of cut-offs $\nu\left(\omega, \alpha,\left\{b_{m}\right\}\right), C_{2}$ is constant.

What about $C_{3}$ ? To determine its fate, we must find the change in the quantity $\mathcal{D}_{2}$ [recall Eq. (41)]. After substituting $\alpha$ for $(2 / W)$, we follow essentially the same path that we blazed in determining the change in $K_{\mathrm{c}}(0)$ and find that the change in $K_{\mathrm{c}}(\alpha x)$ is given by the formula

$$
\delta K_{\mathrm{c}}(\alpha x)=\frac{1}{2 \pi} \sum_{m=1}^{M} b_{m}(m-1) !\left[\frac{1}{(1+i x)^{m}}+\text { c.c. }\right] .
$$

Employing this, we can break the integral on the right side of Eq. (41) into two parts, the first of which is from 0 to $\psi^{1-\epsilon}$, where $0<\epsilon<1$ and $\psi=\left(W / U_{2}\right) \gg 1$. In this interval, the contribution from the entire term proportional to $e^{-\pi K_{\mathrm{c}}(\alpha x)}$ can be shown to be zero in the limit $\psi \rightarrow \infty$. In the remainder, $\delta K_{\mathrm{c}}(\alpha x)$ is on the order of $1 / x^{2}$, which implies that the correction due to the generalization of $\nu\left(\omega, \alpha,\left\{b_{m}\right\}\right)$ is proportional to

$$
\int_{\psi^{1-\epsilon}}^{\beta W / 2} d x \frac{e^{-\pi K_{c}, 0}(2 x / W)}{x^{3}} \leq \int_{\psi^{1-\epsilon}}^{\beta W / 2} \frac{d x}{x^{3}}
$$

which also equals zero in the limit $\psi \rightarrow \infty$. Therefore, $\mathcal{D}_{2}$ is constant, and we have proven that our strong-coupling results are insensitive to varying the cut-offs within the class $\nu\left(\omega, \alpha,\left\{b_{m}\right\}\right)$.

The values of $\mathcal{D}_{1}$ and $\mathcal{D}_{2}$ can be shown to be similarly unaltered when we switch from the exponential cut-off to a Gaussian:

$$
\nu_{G}(\omega, \alpha)=e^{-\frac{\pi}{4} \alpha^{2} \omega^{2}} .
$$

Solving for $e^{-\pi K_{\mathrm{c}}(0)}$ with this weight function, one discovers that

$$
e^{-\pi K_{c, G}(0)}=\frac{e^{\gamma / 2}}{\sqrt{\pi}} \alpha U_{2},
$$

where $\gamma$ is once again the Euler-Mascheroni constant. The normalization coefficient $\mathcal{A}_{G}$ has not been solved for analytically. However, starting from Eq. (47), one finds that

$$
\begin{aligned}
\left|\mathcal{A}_{G}\right|^{-2}=\pi \alpha \int_{0}^{\infty} d x & \cos \left[\frac{\pi}{2} \operatorname{Erf}(x / 2)\right] \\
& \times e^{\frac{i \sqrt{\pi}}{2}} \int_{0}^{x} d y e^{-y^{2} / 4} \operatorname{Erf}(i y / 2)
\end{aligned}
$$

where $\operatorname{Erf}(x)=(2 / \sqrt{\pi}) \int_{0}^{x} d t e^{-t^{2}}$ is the error function. It has been confirmed numerically that through at least 12 digits the product $\mathcal{D}_{1, G}=\left|\mathcal{A}_{G}\right|^{2} e^{-\pi K_{\mathrm{c}, G}(0)}$ agrees with the exponential cut-off. By arguments similar to those used for the class of cut-off functions studied above, it has also been shown that in the limit $\psi \rightarrow \infty$, the integral $\mathcal{D}_{2, G}$ is the same as for the exponential. The coefficients in Eq. (38) are again unaltered, and it seems reasonable to suppose that the invariance is general.

\section{B. Insensitivity to Fermionic Filling Fraction}

Thus, it appears fairly certain that modifying the highenergy density of states in the bosonized theory does not affect the results of Sec. III. Nevertheless, having solved the weak-coupling model for the general case of a fermionic system not necessarily at half-filling and having seen that, when expressed as functions of the tunneling amplitude, both the conductance and the fractional peak splitting depend upon the filling fraction, one might wonder what happens to the strong-coupling results when one begins with a fermionic system that is not necessarily at half-filling. Since Luttinger-style bosonization assumes symmetry between occupied and empty states, such a system can only be properly bosonized after the asymmetric fermion states have been integrated out. For example, if the system is below half-filling $[F<(1-F)]$ and the zero of energy is at the Fermi surface $\left(\epsilon_{F}=0\right)$, the fermionic single-particle states with energies between $F W$ and $(1-F) W$ must be integrated out, leaving a symmetric effective theory with single-particle energies ranging from $-F W$ to $F W$. Only after this symmetrization can the theory be bosonized without losing knowledge of the fermionic filling fraction $F$. 
The task before us, therefore, is to "symmetrize" the fermionic theory that lies behind the bosonized action of Eq. (17). The archetypal fermionic Hamiltonian consists of the usual three parts: the single-particle kinetic energies, the multiparticle potential energy, and the backscattering barrier. The Hamiltonian therefore takes the following form:

$$
\begin{aligned}
H & =H_{K}+H_{C}+H_{B}, \\
H_{K} & =\sum_{j=1}^{2} \sum_{\sigma} \sum_{k} \xi_{k} c_{j k \sigma}^{\dagger} c_{j k \sigma}, \\
H_{C} & =U_{2}(\hat{n}-\rho / 2)^{2}, \\
H_{B} & =\sum_{\sigma} \sum_{k_{1} k_{2}} v\left(c_{2 \mathbf{k}_{\mathbf{2}} \sigma}^{\dagger} c_{1 \mathbf{k}_{\mathbf{1}} \sigma}+\text { H.c. }\right),
\end{aligned}
$$

where $\xi_{k}=\hbar v_{F} k$ and $j$ is the index that distinguishes between right-movers $(j=1)$ and left-movers $(j=2)$.

The operator $\hat{n}$ is now somewhat more complicated than in the weak-coupling theory. In its simplest form, it can be written as

$$
\hat{n}=\frac{1}{2} \sum_{j=1} \int d x[\Theta(x)-\Theta(-x)] \psi_{j}^{\dagger}(x) \psi_{j}(x),
$$

where $\Theta(x)$ is the Heaviside step function and $\psi_{j}$ is the annihilation operator in position space for a right-moving $(j=1)$ or a left-moving $(j=2)$ fermion. After writing the components of the integrand in the momentum representation and integrating over $x$, one finds that, for a one-dimensional system of length $L$,

$$
\hat{n}=\frac{-i}{L} \sum_{j} \sum_{\sigma} \sum_{k_{1} k_{2}} \frac{c_{j k_{2} \sigma}^{\dagger} c_{j k_{1} \sigma}}{k_{2}-k_{1}}\left(1-\delta_{k_{1}, k_{2}}\right),
$$

which is equivalent to the integral version obtained by Matveev 19 from the observation that $d \hat{n} / d t$ equals the current operator at $x=0$, the point of "division" between the two dots. (This point is, of course, not entirely well-defined in the limit $g \rightarrow 1$.)

The above equations for the Hamiltonian and number operator are presented as discrete sums. For future reference in implementing the symmetrization of the theory, we write the components of our Hamiltonian in integral form:

$$
\begin{aligned}
& H_{K}=\left(\frac{\hbar v_{F}}{\delta}\right) \sum_{j=1}^{2} \int d k \xi_{k} c_{j k \sigma}^{\dagger} c_{j k \sigma}, \\
& H_{C}=U_{2}(\hat{n}-\rho / 2)^{2} \\
& H_{B}=v\left(\frac{\hbar v_{F}}{\delta}\right) \sum_{\sigma} \int d k_{1} \int d k_{2}\left(c_{2 \mathbf{k}_{\mathbf{2}} \sigma}^{\dagger} c_{1 \mathbf{k}_{\mathbf{1}} \sigma}+\text { H.c. }\right)
\end{aligned}
$$

where $\delta$ is the level-spacing for the one-dimensional system $\left(\delta=2 \pi \hbar v_{F} / L\right)$ and

$$
\hat{n}=\frac{-i}{2 \pi} \sum_{j} \sum_{\sigma} \mathcal{P} \int d k_{1} \int d k_{2} \frac{c_{j k_{2} \sigma}^{\dagger} c_{j k_{1} \sigma}}{k_{2}-k_{1}} .
$$

For the fermionic strong-coupling model of Eq. (57), calculation of the channel conductance between the dots proceeds along the same lines as for weak coupling (see Ref. 4). In fact, since the density of states is constant in both theories, setting $U_{2}=0$ - the first step in the conductance calculation - renders them essentially identical, the only differences being in the last term, where $t$ has been replaced by $v$ and the index $i$ for dot1 or dot- 2 fermions has been replaced by the index $j$ for right-movers or left-movers. Accordingly, unlike the weak-tunneling term $H_{T}$, the perturbation $H_{B}$ scatters fermions backward instead of transporting them forward and therefore causes a reduction in the conductance of the unperturbed system. Recalling the size of the conductance induced by $H_{T}$ in the weak-coupling model, it is not hard to see that the channel conductance in the strong-coupling model is given by

$$
g=1-\frac{4 \chi}{\left|1+(1+i \eta)^{2} \chi\right|^{2}},
$$

where $\chi=(\pi v / \delta)^{2}$ and $\eta=(1 / \pi) \ln [F /(1-F)]$. As in the weak-coupling theory, the result becomes troublesome as $\chi$ becomes large. However, we should be able to trust its testimony that the filling fraction does not affect the interdot conductance through second-order in $(\pi v / \delta)$.

This is all we need to know, for $(\pi v / \delta)$ can be straightforwardly written in terms of our previous strongcoupling parameter $\tilde{V}$. The relation is $\tilde{V}=2(\pi v / \delta)$, and it follows that $\chi=(\tilde{V} / 2)^{2}$. We recover the leading-order result of Eq. (20) and see that, to order $\tilde{V}^{2}$, the channel conductance $g$ is independent of the fermionic filling fraction $F$. If we can likewise show that the relation between $\tilde{V}$ and the differential energy shift

$$
\delta \Delta(\rho)=\left[\Delta_{\text {str }}(\rho)-\Delta_{\text {str }}(0)\right]
$$

does not depend on the filling fraction $F$, we will know that the same is true for our final strong-coupling result, the expression for $f(g)$ in Eq. (38).

To prove $\delta \Delta$ 's invariance with respect to $F$, we symmetrize the fermionic theory through a renormalization in which we integrate out all single-particle states at an energy distance of $W^{\prime} / 2$ or more from the Fermi surface, where $U_{2} \ll W^{\prime} \ll W$. The resulting symmetric theory with bandwidth $W^{\prime}$ can be bosonized without further qualm. However, as renormalization generates terms that are not present in the original Hamiltonian, we must check to see what relevant effects these have upon the low-energy theory. We must also keep track of any contributions to $\delta \Delta$ that arise from the high-energy degrees of freedom alone.

Before we go about doing this, a comment on our approach is in order. One might view the proposed renormalization as occurring in two distinct stages: first, we integrate out the asymmetric particle-hole states; then, 
we integrate both particle and hole states down to energy $W^{\prime}$. Since all that we will need to consider are the general scaling properties of the terms generated during the renormalization process, the distinction between the stages is of no importance and is henceforth ignored.

The argument resumes. Since our interest is in the Coulomb blockade, the renormalization scheme we use is designed to leave the Coulombic interaction term $H_{C}$ unchanged. After wave-vectors between the original wavevector cut-off $\Lambda$ and the new wave-vector cut-off $\Lambda / b$ (where $b>1$ ) have been integrated out, the theory is re-scaled by writing it in terms of a new set of wavevectors $k_{b}=b k$. Invariance of $H_{C}$ is achieved by rescaling the fermion creation and annihilation operators as well: $c_{j k_{b} \sigma}^{\dagger}=b^{-1 / 2} c_{j k \sigma}^{\dagger}$. [One might prefer to say that the coherent-state Grassman variables that correspond to the operators are re-scaled (see Ref. 38).] The effect of renormalization upon the parameters $\left(\hbar v_{F} / \delta\right), U_{2}$, and $v\left(\hbar v_{F} / \delta\right)$ of Eq. (57) is as follows:

$$
\begin{aligned}
{\left[\frac{\hbar v_{F}}{\delta}\right]^{\prime} } & =b^{-1}\left[\frac{\hbar v_{F}}{\delta}\right], \\
{\left[U_{2}\right]^{\prime} } & =U_{2}, \\
{\left[v\left(\hbar v_{F} / \delta\right)\right]^{\prime} } & =b^{-1}\left[v\left(\hbar v_{F} / \delta\right)\right] .
\end{aligned}
$$

The backscattering $H_{B}$ is revealed to be dangerously irrelevant. Though it scales like an irrelevant term, we cannot safely set it to zero as we know from Eq. (33) that the energy shift is singular as $v \rightarrow 0$.

In addition to rescaling the terms in the original Hamiltonian, renormalization generates terms of its own. It is not hard to see that all but the new backscattering terms are irrelevant. The original Hamiltonian consists of the kinetic energy $H_{K}$, a two-body interaction $H_{C 2}$, a onebody interaction $H_{C 1}$, and a backscattering term $H_{B}$. $H_{C 2}$ and $H_{C 1}$ are normal-ordered operators given by the following formulas:

$$
\begin{aligned}
H_{C 2}=\frac{-U_{2}}{(2 \pi)^{2}} \sum_{j_{1}, j_{2}} & \sum_{\sigma_{1}, \sigma_{2}} \mathcal{P} \int d k_{1} \ldots d k_{4} \\
& \times \frac{c_{j_{2} k_{4} \sigma_{2}}^{\dagger} c_{j_{1} k_{2} \sigma_{1}}^{\dagger} c_{j_{1} k_{1} \sigma_{1}} c_{j_{2} k_{3} \sigma_{2}}}{\left(k_{4}-k_{3}\right)\left(k_{2}-k_{1}\right)}, \\
H_{C 1}=-\rho U_{2} \hat{n}+ & \frac{-U_{2}}{(2 \pi)^{2}} \sum_{j} \sum_{\sigma} \mathcal{P} \int d k_{1} \int d k_{2} \frac{c_{j k_{2} \sigma}^{\dagger} c_{j k_{1} \sigma}}{k_{2}-k_{1}} \\
& \times\left(\ln \left|\frac{\Lambda-k_{1}}{\Lambda+k_{1}}\right|-\ln \left|\frac{\Lambda-k_{2}}{\Lambda+k_{2}}\right|\right), \quad \text { (65) }
\end{aligned}
$$

where $\Lambda=W / \hbar v_{F} . H_{C 2}, H_{C 1}$, and $H_{B}$ can be represented by Feynman graphs [see Fig. 4(a)], which can then be connected to construct the terms that renormalization adds to the Hamiltonian. As usual, the internal lines of the second-generation graphs carry only high-energy momenta which lie within the shell of wave-vectors that are integrated out. (a)

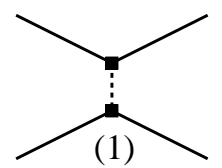

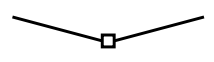

(2)

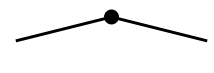

(3)

(b)

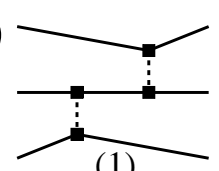

(1)

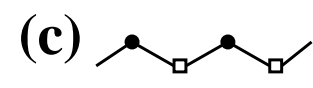

(1)

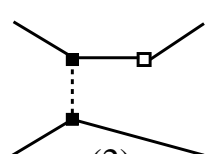

(2)

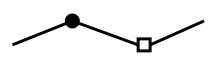

(2)

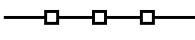

(3)

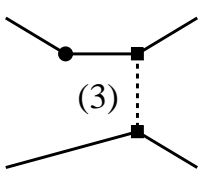

(d)

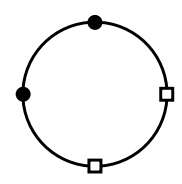

FIG. 4. Feynman diagrams for integrating out single-particle energies a distance greater than $W^{\prime}$ from the Fermi surface in the fermionic version of the strong-coupling theory. (a) The three building-block Feynman graphs. Diagram 1 corresponds to the two-body Coulomb interaction $H_{C 2}$. Diagram 2 corresponds to the one-body Coulomb term $H_{C 1}$. Diagram 3 represents the backscattering $H_{B}$. (b) Second-generation $m$-body graphs constructed by contracting $H_{C 2}$ 's and $H_{C 1}$ 's. These terms are all irrelevant to the low-energy theory, scaling to zero under renormalization. (c) Second-generation graphs formed from combinations of $H_{B}, H_{C 2}$, and $H_{C 1}$. Terms such as Diagram 1 that contain an even number of $H_{B}$ 's are irrelevant under renormalization. Diagrams 2 and 3 involve odd numbers of $H_{B}$ 's and are therefore dangerously irrelevant. Nevertheless, they are negligible in size compared to corresponding low-energy graphs and therefore can be safely discarded. (d) An example of a closed diagram used to calculate the contribution to the energy shift from the degrees of freedom that correspond to single-particle energies more than $W^{\prime}$ from the Fermi surface.

Given such rules for making second-generation terms, one can deduce that, whenever one creates a new term by connecting lines emanating from the $H_{C 2}$ and $H_{C 1}$ graphs [see Fig. 4(b) for examples], one picks up a scaling factor of $b^{-1}$. For example, Diagram 2 of Fig. 4(b) represents a two-body interaction produced by contracting one $H_{C 2}$ with one $H_{C 1}$. This new interaction term is similar to $H_{C 2}$ except that the denominator contains only one power of $\left(k_{4}-k_{3}\right)$ or $\left(k_{2}-k_{1}\right)$ and, consequently, is less singular than $H_{C 2}$, which is fixed under rescaling. Thus, the second-generation term must shrink under renormalization. Indeed, all such graphs formed from contracting the Coulombic interaction terms are similarly irrelevant and scale to zero under renormalization. They can be 
ignored in the effective theory. We should expect this result. Otherwise, our Coulomb blockade model would probably never have been useful at all.

As for graphs that involve the backscattering term $H_{B}$ [see Fig. 4(c)], we need only consider these to order $v^{2}$, for we go no further in calculating $f(g)$. Depending upon how many Coulombic interaction terms are introduced, the second-generation graphs that contain $H_{B}$ all scale down by at least a factor of $b^{-1}$. Consequently, all but those which contribute to low-energy backscattering are irrelevant. Thus, we can drop graphs such as Diagram 1 of Fig. 4(c) that contain an even number of $H_{B}$ 's. Graphs containing an odd number of $H_{B}$ 's are dangerously irrelevant but can ultimately be ignored because they are negligible compared to the corresponding graphs that can be constructed from the low-energy portions of the original $H_{C 2}, H_{C 1}$, and $H_{B}$. Diagrams 2 and 3 of Fig. 4(c), for example, are of order $v\left(U_{2} / W^{\prime}\right)$. If we had renormalized down to $W^{\prime \prime}$, where $U_{2} \ll W^{\prime \prime} \ll W^{\prime}$, we would have found the corresponding graphs to be of order $v\left(U_{2} / W^{\prime \prime}\right)$. The contribution from energies above $W^{\prime}$ is therefore seen to be merely perturbative in relation to the contribution from energies between $W^{\prime \prime}$ and $W^{\prime}$. The conclusion is that we can drop the parts of the graphs produced by integrating over energies greater than $W^{\prime}$. Returning to our original renormalization down to $W^{\prime}$, we see that the graphs produced here have been shown to be negligible. The argument that the symmetrizing renormalization does not cause any significant changes in the low-energy Hamiltonian is complete.

Having disposed of the concern that the process of symmetrization might leave us with important new lowenergy terms, we now show that any constant terms produced are similarly insignificant. Such constant terms correspond to closed diagrams constructed from the original Feynman graphs. Since all lines are internal, they all represent the propagation of high-energy excitations. There are obviously an infinite number of closed diagrams. Fortunately, we can limit our attention to a certain subset. We need not concern ourselves with diagrams involving less than two $H_{C 1}$ graphs: diagrams with only one $H_{C 1}$ graph must sum to zero as $\Delta(\rho)$ is even in $\rho$; diagrams with zero $H_{C 1}$ graphs cannot contribute to the differential energy shift $\delta \Delta$. Similarly, in any pertinent closed graph, $H_{B}$ must appear a nonzero and even number of times. It cannot be absent as terms that do not include it shift all relevant ground-state energies equally and are therefore unimportant. Furthermore, in any closed graph, it must appear an even number of times because $H_{B}$ is the only term that exchanges rightand left-movers. Thus, all the diagrams we need consider consist of a nonzero and even number of $H_{B}$ 's, at least two $H_{C 1}$ 's, and an arbitrary number of $H_{C 2}$ 's [see Fig. 4(d) for a canonical example].

Each such diagram corresponds to a number of timeordered terms in Rayleigh-Schrödinger perturbation theory. For a Feynman diagram with $r$ internal lines, the associated Rayleigh-Schrödinger terms have $r$ integrations over momenta and $(r-1)$ propagators with denominators linear in the momenta. If the $H_{C 2}$ graph appears $m_{2}$ times in the Feynman diagram and the $H_{C 1}$ graph appears $m_{1}$ times, there are $m=\left(2 m_{2}+m_{1}\right) \geq 2$ additional denominators linear in the momenta, which have their origin in the wave-vector denominator of $\hat{n}$ [recall Eqs. (61) and (65)]. The propagator denominators are always on the order of $W^{\prime}$ or greater. The $\hat{n}$ denominators are of the form $\left(k-k^{\prime}\right)$, where $k$ and $k^{\prime}$ are both in the high-energy wave-vector shell. Thus, these denominators can go to zero. However, the contribution from the regions where they become zero is negligible, the somewhat simplified explanation being that, when one of them goes to zero, the rest of the integrand can be treated as essentially constant, and we have

$$
\mathcal{P} \int_{-\epsilon \Lambda^{\prime}}^{\epsilon \Lambda^{\prime}} \frac{1}{k}\left[1+\mathcal{O}\left(\frac{\hbar v_{F} k}{W^{\prime}}\right)\right]=\mathcal{O}(\epsilon),
$$

where $\Lambda^{\prime}=W^{\prime} / \hbar v_{F}$ and the constant $\epsilon \ll 1$. It follows that contributions to the overall result only come when the $\hat{n}$ denominators are themselves of order $W^{\prime} / \hbar v_{F}$.

As a result, what remains is a nonsingular integration over $r$ momenta of an integrand that is proportional to $\left[\bar{k}_{1} \ldots \bar{k}_{(r-1+m)}\right]^{-1}$, where the $\bar{k}_{i}$ are linear in the momenta over which we integrate. Noting that the only other momentum dependence comes from the logarithmic term of $H_{C 1}$, we see that, in energy units, the result of the integration is of order $\left(1 / W^{\prime}\right)^{m-1}$. We now multiply the result of our integration by the various factors of $U_{2}, v$, and $\delta$ that stand aside the integral. For a closed diagram in which $H_{B}, H_{C 2}$, and $H_{C 1}$ appear $j, m_{2}$, and $m_{1}$ times, respectively, the contribution to the energy shift is readily seen to be of order $U_{2}(v / \delta)^{2 j}\left(U_{2} / W^{\prime}\right)^{m-1}$, where $m=\left(2 m_{2}+m_{1}\right) \geq 2$. As $\left(U_{2} / W^{\prime}\right) \ll 1$ and the overall energy shift is of order $U_{2}$, these terms are negligible.

Thus, at least to order $v^{2}$, integrating out all particle and hole excitations at distances greater than $W^{\prime} / 2$ from the Fermi surface produces neither relevant new terms in the low-energy Hamiltonian nor significant constant contributions to the differential energy shift. As what remains is a fermionic theory at half-filling, the result for $f(g)$ in Sec. III is unaffected by possible "high-energy" deviations from this condition, an important property if we wish to compare our predictions with empirical data. We would hope that a similarly universal solution for $f(g)$ could be found to higher orders in $(1-g)$. However, if the formula for the interdot conductance [recall Eq. (622] is correct to some non-leading order, such overall independence of the filling fraction must - as in the weak-coupling limit - come through cancellation of the separate filling-fraction dependences of the conductance and the energy shift when one is expressed in terms of the other. If this were shown to be true, we would see once again that the interdot conductance $g$ and not the bare matrix element for tunneling or reflection is the correct parameter to achieve a universal description of the coupling dependence of a double-dot Coulomb blockade. 


\section{CONCLUSION}

The present paper substantially improves the results of earlier theoretical work on the Coulomb-hlsckade peaksplitting for two coupled quantum dots 6 making an important contribution to the growing body of theoretical and experimental work on such coupled-dot systems 3 , $30,23-35$. By extending the weak-coupling theory to second order in $g$ for arbitrary $N_{\mathrm{ch}}$, it has shown how the small- $N_{\text {ch }}$ theory crosses over to the large- $N_{\text {ch }}$ theory in the neighborhood of $N_{\mathrm{ch}} \approx 10$. Furthermore, it has demonstrated that, at least for the leading two terms in the weak-coupling theory, the channel conductance $g$ is the "correct" parameter to use in constructing a theory for the peak splitting that is universal in the sense that it is does not depend on the high-energy band structure. Finally, this paper has made the $N_{\mathrm{ch}}=2$ theory both stronger and broader-broader in that the sub-leading term is calculated; stronger in that the leading and subleading terms for strong-coupling are confirmed to be insensitive to the manner in which the high-energy cut-off is taken. Thus, the paper has made more plausible efforts to connect weak- and strong-coupling behaviors and to compare theoretical resilts, with the data from recent two-channel experiments. 2 , 3.10

\section{ACKNOWLEDGMENTS}

The authors are grateful for helpful conversations with F. R. Waugh, R. M. Westervelt, C. H. Crouch, C. Livermore, A. L. Moustakas, S. H. Simon, and S. Ramanathan. J. M. G. thanks the United States Air Force for financial support. This work was also supported by the NSF through the Harvard Materials Research Science and Engineering Center, Grant No. DMR94-00396.

\section{APPENDIX A: DETAILS OF THE WEAK-COUPLING CALCULATION}

As described in Sec. II, the procedure in evaluating the fourth-order energy shift is to calculate the $\left(N_{\mathrm{ch}}\right)^{2}$ and $N_{\mathrm{ch}}$ terms separately. Calculation of the $\left(N_{\mathrm{ch}}\right)^{2}$ terms is facilitated by rewriting them in terms of two energy variables instead of four. Calculation of the $N_{\mathrm{ch}}$ terms is made easier by differentiating twice with respect to $\rho$ while performing the integrations over energy and then integrating twice with respect to $\rho$ at the end. Terms that are constant or linear with respect to $\rho$ cancel in the final result, the relative energy shift $\left(\Delta_{0}-\Delta_{\rho}\right)$, so we have not lost useful information as a result of the double differentiation.

As mentioned in Sec. II, the "wrinkle" in these computations, the appearance of integrals of the form

$$
\mathcal{P} \int_{0}^{R \psi} d x \frac{\ln (x+B)}{x+A},
$$

is resolved by Taylor-expanding the logarithm about $(x+A)$ for $(B-A)<|x+A|$ and about $(B-A)$ for $(B-A)>$ $|x+A|$. For $A<0$, one first breaks the integral into the intervals $(0,|A|-\epsilon)$ and $(|A|+\epsilon, R \psi)$. After this, one proceeds as usual. The results are

$$
\begin{aligned}
& \mathcal{P} \int_{0}^{R \psi} d x \frac{\ln (x+B)}{x+A}=\frac{1}{2} \ln ^{2}(R \psi+A)-\frac{1}{2} \ln ^{2} A \\
& +\sum_{n=1}^{\infty} \frac{(-1)^{n+1}}{n^{2}}\left[\left(\frac{B-A}{A}\right)^{n}-\left(\frac{B-A}{R \psi+A}\right)^{n}\right] \\
& \text { for } 0<(B-A)<A<(R \psi+A) \text {, } \\
& =\frac{1}{2} \ln ^{2}(R \psi+A)-\ln (B-A) \ln A+\frac{1}{2} \ln ^{2}(B-A)+\frac{\pi^{2}}{6} \\
& -\sum_{n=1}^{\infty} \frac{(-1)^{n+1}}{n^{2}}\left[\left(\frac{A}{B-A}\right)^{n}+\left(\frac{B-A}{R \psi+A}\right)^{n}\right] \\
& \text { for } 0<A<(B-A)<(R \psi+A) \text {, } \\
& =\ln (B-A)[\ln (R \psi+A)-\ln A] \\
& +\sum_{n=1}^{\infty} \frac{(-1)^{n+1}}{n^{2}}\left[\left(\frac{R \psi+A}{B-A}\right)^{n}-\left(\frac{A}{B-A}\right)^{n}\right] \\
& \text { for } 0<A<(R \psi+A)<(B-A) \text {, }
\end{aligned}
$$




$$
\begin{gathered}
=\frac{1}{2} \ln ^{2}(R \psi-|A|)-\ln (B+|A|) \ln |A|+\frac{1}{2} \ln ^{2}(B+|A|)+\frac{\pi^{2}}{6} \\
+\sum_{n=1}^{\infty} \frac{1}{n^{2}}\left(\frac{|A|}{B+|A|}\right)^{n}-\sum_{n=1}^{\infty} \frac{(-1)^{n+1}}{n^{2}}\left(\frac{B+|A|}{R \psi-|A|}\right)^{n} \\
\quad \text { or } A<0<(B+|A|)<(R \psi-|A|), \\
=\ln (B+|A|)[\ln (R \psi-|A|)-\ln |A|] \\
+\sum_{n=1}^{\infty} \frac{1}{n^{2}}\left(\frac{|A|}{B+|A|}\right)^{n}+\sum_{n=1}^{\infty} \frac{(-1)^{n+1}}{n^{2}}\left(\frac{R \psi-|A|}{B+|A|}\right)^{n} \\
\text { for } A<0<(R \psi-|A|)<(B+|A|) .
\end{gathered}
$$

These five integrals are all we need. In confirming that he solution for $(B-A)<(R \psi+A)$ evolves continuously into that for $(R \psi+A)<(B-A)$, it is useful to recognize 18 that

$$
\sum_{n=1}^{\infty} \frac{(-1)^{n+1}}{n^{2}}=\frac{\pi^{2}}{12}
$$

Having equipped ourselves to smooth the "wrinkles," we can proceed with a fuller description of calculation of the fourth-order terms. The $\left(N_{\mathrm{ch}}\right)^{2}$ calculation is reviewed first. An illustrative segment of the $N_{\mathrm{ch}}$ calculation follows.

In Sec. II, it was remarked that each of the $\left(N_{\mathrm{ch}}\right)^{2}$ terms could be written in terms of two energy variables $\left(\epsilon_{I}=\epsilon_{3}-\epsilon_{1}, \epsilon_{4}-\epsilon_{2}\right)$ instead of the original four. The "cost" of this conversion is the appearance of a nontrivial density of states:

$$
\int_{0}^{\epsilon_{F}} d \epsilon_{1} \int_{0}^{\epsilon_{F}} d \epsilon_{2} \int_{\epsilon_{F}}^{W} d \epsilon_{3} \int_{\epsilon_{F}}^{W} d \epsilon_{4} h\left(\epsilon_{3}-\epsilon_{1}, \epsilon_{4}-\epsilon_{2}\right)=\int_{0}^{\epsilon_{F}} d \epsilon_{\mathrm{I}} \nu\left(\epsilon_{\mathrm{I}}\right) \int_{0}^{\epsilon_{F}} d \epsilon_{\mathrm{II}} \nu\left(\epsilon_{\mathrm{II}}\right) h\left(\epsilon_{\mathrm{I}}, \epsilon_{\mathrm{II}}\right),
$$

where $\nu(\epsilon)$ is the density of states. For a system at or below half-filling,

$$
\nu(\epsilon)= \begin{cases}\epsilon & \text { for } 0 \leq \epsilon<\epsilon_{F}, \\ \epsilon_{F} & \text { for } \epsilon_{F} \leq \epsilon<\left(W-\epsilon_{F}\right), \\ (W-\epsilon) & \text { for }\left(W-\epsilon_{F}\right) \leq \epsilon<W .\end{cases}
$$

(We need not worry about a system above half-filling as such as system can be mapped to one below half-filling through an exchange of particles and holes.)

Using the new variables $\epsilon_{\mathrm{I}}$ and $\epsilon_{\mathrm{II}}$, we can sum the integrands for the $\left(N_{\mathrm{ch}}\right)^{2}$ terms shown in Fig. 2 (the others are obtained by taking $\rho \rightarrow-\rho$ ). If drop the common factor $-N_{\mathrm{ch}}(t / \delta)^{4} U_{2}$, the result is the following:

$$
\begin{aligned}
h_{\mathrm{tot}}= & \frac{-2}{\left[\epsilon_{\mathrm{I}}+U_{2}(1-\rho)\right]^{2}\left[\epsilon_{\mathrm{II}}+U_{2}(1-\rho)\right]\left[\epsilon_{\mathrm{II}}+\epsilon_{\mathrm{I}}+U_{2}(4-2 \rho)\right]} \\
& +\frac{2}{\left[\epsilon_{\mathrm{I}}+U_{2}(1-\rho)\right]^{2}\left[\epsilon_{\mathrm{II}}+U_{2}(1+\rho)\right]\left[\epsilon_{\mathrm{II}}+\epsilon_{\mathrm{I}}\right]} .
\end{aligned}
$$

It is not hard to show that

$$
\int_{\epsilon_{F}}^{W} d \epsilon_{\mathrm{I}} \int_{\epsilon_{F}}^{W} d \epsilon_{\mathrm{II}}\left[h_{\mathrm{tot}}\left(\epsilon_{\mathrm{I}}, \epsilon_{\mathrm{II}}, \rho\right)-h_{\mathrm{tot}}\left(\epsilon_{\mathrm{I}}, \epsilon_{\mathrm{II}}, 0\right)\right]=0
$$

in the limit $\psi=W / U_{2} \rightarrow \infty$. Accordingly, we need only calculate

$$
\int_{0}^{\epsilon_{F}} d \epsilon_{\mathrm{I}} \int_{0}^{\epsilon_{F}} d \epsilon_{\mathrm{II}} h_{\mathrm{tot}}\left(\epsilon_{\mathrm{I}}, \epsilon_{\mathrm{II}}, \rho\right)
$$

The process of evaluating this double integral is lengthy but straightforward. The only "wrinkles" that appearintegrals of the form of Eq. A1 - are no longer problematic. The end result is Eq. (9) of Sec. II.

We now move to consideration of the fourth-order terms linear in the number of conducting channels. Recall that the $\left(N_{\mathrm{ch}}\right)^{2}$ terms were added before the integrations over energy were performed. This order of tasks is reversed for 
the $N_{\mathrm{ch}}$ terms, the computation of which revolves primarily about finding a favorable permutation of the operations of differentiating and integrating with respect to $\rho$, integrating with respect to the $i$ th energy variable, and integrating by parts. Consequently, perhaps the best way to describe the derivation of the $N_{\mathrm{ch}}$ contribution is to walk through the computation of a single illustrative term. After seeing the methodology employed in calculating this term, the tireless reader should have little difficulty in computing the rest.

The representative term we choose is that corresponding to Diagram 2 of Fig. 2(b). This term involves an exchange of a pair of electrons and, consequently, picks up an "exchange" minus sign. The diagram depicts the following sequence of events:

I. Electron 1 tunnels from dot 1 to dot 2 , going from a single-particle state with kinetic energy $\epsilon_{1}$ to one with kinetic energy $\epsilon_{3}$. The energy of the resulting double-dot state relative to that of the unperturbed ground state is $\left[\epsilon_{3}-\epsilon_{1}+U_{2}(1-\rho)\right]$.

II. Electron 2 tunnels from dot 1 to dot 2, going from a single-particle state with kinetic energy $\epsilon_{2}$ to one with kinetic energy $\epsilon_{4}$. The system's energy relative to the unperturbed ground state is now $\left[\epsilon_{4}+\epsilon_{3}-\epsilon_{2}-\epsilon_{1}+2 U_{2}(2-\rho)\right]$.

III. Electron 2 tunnels back to dot 1, settling into the initial single-particle state of Electron 1. The ensuing relative system energy is $\left[\epsilon_{3}-\epsilon_{2}+U_{2}(1-\rho)\right]$.

IV. Electron 1 tunnels back to dot 1, settling into Electron 2's initial single-particle state. The unperturbed ground state has been recovered.

With all the intermediate-state energies known, it is easy to write down the contribution to the fourth-order energy shift:

$$
\begin{aligned}
\Delta_{N_{\mathrm{ch}}, 2}^{(4)}(\rho)=t^{4} \sum_{\sigma} & \sum_{\epsilon_{1}, \epsilon_{2}} \sum_{\epsilon_{3}, \epsilon_{4}} \frac{1}{\left[\epsilon_{3}-\epsilon_{2}+U_{2}(1-\rho)\right]} \\
& \times \frac{1}{\left[\epsilon_{4}+\epsilon_{3}-\epsilon_{2}-\epsilon_{1}+2 U_{2}(2-\rho)\right]} \times \frac{1}{\left[\epsilon_{3}-\epsilon_{1}+U_{2}(1-\rho)\right]} .
\end{aligned}
$$

The sums over $\epsilon_{1}$ and $\epsilon_{2}$ extend from 0 to the Fermi energy $\epsilon_{F}$. Those for $\epsilon_{3}$ and $\epsilon_{4}$ go from $\epsilon_{F}$ to the bandwidth $W$. The sum over the channel index $\sigma$ results from the fact that Electrons 1 and 2 can share any one of the $N_{\mathrm{ch}}$ tunneling channels. Though the formula contains such unphysical terms as that for which $\epsilon_{1}=\epsilon_{2}$, such terms are down by factors of the level spacing $\delta$ divided by $\delta / F W$ or $\delta /(1-F) W$, and their inclusion has no effect in the limit $W / \delta \rightarrow \infty$.

Accordingly, we can cease worrying about these terms, for we assume that $\delta \ll U_{2} \ll W$, a postulate that permits us to work in the continuum limit, replacing the sums in Eq. (A6) by integrals:

$$
\begin{aligned}
\Delta_{N_{\mathrm{ch}}, 2}^{(4)}(\rho)=N_{\mathrm{ch}} & \left(\frac{t}{\delta}\right)^{4} \int_{0}^{\epsilon_{F}} d \epsilon_{1} \int_{0}^{\epsilon_{F}} d \epsilon_{2} \int_{\epsilon_{F}}^{W} d \epsilon_{3} \int_{\epsilon_{F}}^{W} d \epsilon_{4} \frac{1}{\left[\epsilon_{3}-\epsilon_{2}+U_{2}(1-\rho)\right]} \\
& \times \frac{1}{\left[\epsilon_{4}+\epsilon_{3}-\epsilon_{2}-\epsilon_{1}+2 U_{2}(2-\rho)\right]} \times \frac{1}{\left[\epsilon_{3}-\epsilon_{1}+U_{2}(1-\rho)\right]} .
\end{aligned}
$$

These integrals can be rewritten in terms of dimensionless variables $x_{i}$ :

$$
x_{i}=\left\{\begin{array}{lll}
\frac{\epsilon_{F}-\epsilon_{i}}{U_{2}} & \text { for } i=1 \text { or } 2 \\
\frac{\epsilon_{i}-\epsilon_{F}}{U_{2}} & \text { for } i=3 \text { or } 4 .
\end{array}\right.
$$

With this choice of integration variables, it becomes clear that $\Delta_{N_{\mathrm{ch}}, 2}^{(4)}(\rho)$ is linear in $U_{2}$. Specifically, we find that

$$
\begin{aligned}
\Delta_{N_{\mathrm{ch}}, 2}^{(4)}(\rho)= & N_{\mathrm{ch}}\left(\frac{t}{\delta}\right)^{4} U_{2} \times I(\rho, F, \psi), \\
I(\rho, F, \psi)= & \int_{0}^{F \psi} d x_{1} \int_{0}^{F \psi} d x_{2} \int_{0}^{(1-F) \psi} d x_{3} \int_{0}^{(1-F) \psi} d x_{4} \frac{1}{\left[x_{3}+x_{2}+1-\rho\right]} \\
& \quad \times \frac{1}{\left[x_{4}+x_{3}+x_{2}+x_{1}+2(2-\rho)\right]} \times \frac{1}{\left[x_{3}+x_{1}+1-\rho\right]} .
\end{aligned}
$$

All the shuffling of notatation still leaves us confronted with a quadruple integral. Opting to postpone a frontal assault, we try a sidestepping movement, computing the partial derivative with respect to $\rho$ : 


$$
I_{\rho}=\int_{0}^{F \psi} d x_{1} \int_{0}^{F \psi} d x_{2} \int_{0}^{(1-F) \psi} d x_{3} \int_{0}^{(1-F) \psi} d x_{4}\left(\frac{1}{[]^{2}[][]}+\frac{2}{[][]^{2}[]}+\frac{1}{[][][]^{2}}\right)
$$

where the subscript $\rho$ signifies that $I_{\rho}$ is the partial derivative of $I$ with respect to $\rho$ and the brackets on the right-hand side of the equation have the same contents in the same order as those in Eq. (A9). As the third term in the integrand of Eq. A10) differs from the first only by an exchange of the indices 1 and 2, we can drop the third term and double the first. When the enhanced first term is integrated by parts with respect to $x_{2}$, the products are two triple-integral terms and a quadruple-integral term that exactly cancels the second term of Eq. (A10). With the definitions $A_{0}=0$ and $A_{1}=F \psi$, we have

$$
\begin{aligned}
I_{\rho}=2 \sum_{p=0}^{1}( & -1)^{p} \int_{0}^{F \psi} d x_{1} \int_{0}^{(1-F) \psi} d x_{3} \int_{0}^{(1-F) \psi} d x_{4} \frac{1}{\left[x_{3}+A_{p}+1-\rho\right]} \\
& \times \frac{1}{\left[x_{4}+x_{3}+x_{1}+A_{p}+2(2-\rho)\right]} \times \frac{1}{\left[x_{3}+x_{1}+1-\rho\right]} .
\end{aligned}
$$

Having benefited once from differentiation with respect to $\rho$, we try it again. The second derivative of $I$ with respect to $\rho$ has the following form:

$$
I_{\rho \rho}=2 \int_{0}^{F \psi} d x_{1} \int_{0}^{(1-F) \psi} d x_{3} \int_{0}^{(1-F) \psi} d x_{4}\left(\frac{1}{[]^{2}[][]}+\frac{2}{[][]^{2}[]}+\frac{1}{[][][]^{2}}\right),
$$

where the bracket contents correspond - in order of appearance - to those of Eq. (A11). $I_{\rho \rho}$ lacks the convenient symmetry between first and third terms that was so handy before. Nevertheless, integration of the first term by parts with respect to $x_{3}$ still helps. The triple integrals that result cancel the third term and half the middle term, leaving

$$
\begin{aligned}
& I_{\rho \rho}=2 \sum_{p=0}^{1} \sum_{q=0}^{1} \frac{(-1)^{p+q}}{A_{p}+B_{q}+1-\rho} \int_{0}^{F \psi} d x_{1} \int_{0}^{(1-F) \psi} d x_{4} \\
& \times \frac{1}{\left[x_{4}+x_{1}+A_{p}+B_{q}+2(2-\rho)\right]\left[x_{1}+B_{q}+1-\rho\right]} \\
&+2 \sum_{p=0}^{1}(-1)^{p} \int_{0}^{F \psi} d x_{1} \int_{0}^{(1-F) \psi} d x_{3} \int_{0}^{(1-F) \psi} d x_{4} \frac{1}{\left[x_{3}+A_{p}+1-\rho\right]} \\
& \times \frac{1}{\left[x_{4}+x_{3}+x_{1}+A_{p}+2(2-\rho)\right]^{2}\left[x_{3}+x_{1}+1-\rho\right]},
\end{aligned}
$$

where $B_{0}=0$ and $B_{1}=(1-F) \psi$.

We now straightforwardly integrate over $x_{4}$, using the relation

$$
\frac{1}{(x+a)(x+b)}=\frac{1}{b-a}\left(\frac{1}{x+a}-\frac{1}{x+b}\right) .
$$

The result is that

$$
\begin{aligned}
I_{\rho \rho}=2 \sum_{p=0}^{1} \sum_{q=0}^{1} & \sum_{r=0}^{1} \frac{(-1)^{p+q+r+1}}{A_{p}+B_{q}+1-\rho} \int_{0}^{F \psi} d x \frac{\ln \left[x+A_{p}+B_{q}+B_{r}+2(2-\rho)\right]}{x+B_{q}+1-\rho} \\
+2 \sum_{p=0}^{1} \sum_{q=0}^{1} & \frac{(-1)^{p+q}}{A_{p}+B_{q}+3-\rho} \int_{0}^{F \psi} d x_{1} \int_{0}^{(1-F) \psi} d x_{3} \frac{1}{\left[x_{3}+A_{p}+1-\rho\right]} \\
& \times\left(\frac{1}{\left[x_{3}+x_{1}+1-\rho\right]}-\frac{1}{\left[x_{3}+x_{1}+A_{p}+B_{q}+2(2-\rho)\right]}\right) .
\end{aligned}
$$

Recalling Eq. (A1), we see that, as $\psi \rightarrow \infty$, the leading part of the first term in Eq. (A14) goes as $\left[\ln ^{2} \psi /\left(A_{p}+B_{q}+\right.\right.$ $1-\rho)]$ and therefore goes to zero unless $p=q=0$. The same is true for the second term - which upon integration over $x_{1}$ will have a form like that of the first term. Hence, we can eliminate the sums over $p$ and $q$ and, after integrating the second term over $x_{1}$, have 


$$
\begin{aligned}
I_{\rho \rho}= & \frac{2}{1-\rho} \sum_{r=0}^{1}(-1)^{r+1} \int_{0}^{F \psi} d x \frac{\ln \left[x+B_{r}+2(2-\rho)\right]}{x+1-\rho} \\
& +\frac{2}{3-\rho} \sum_{r=0}^{1}(-1)^{r+1} \int_{0}^{(1-F) \psi} d x \frac{\ln \left[\frac{x+A_{r}+1-\rho}{x+A_{r}+2(2-\rho)}\right]}{x+1-\rho} .
\end{aligned}
$$

We recognize that the second term is nontrivial only for $r=0$ and apply Eq. (A1) to do the remaining integrations over $x$. After dropping terms that go to zero as $\psi \rightarrow \infty$, we arrive at the "final" formula for $I_{\rho \rho}$ :

$$
\begin{aligned}
I_{\rho \rho}= & I_{\rho \rho}^{(1)}+I_{\rho \rho}^{(2)}, \\
\left(\frac{1-\rho}{2}\right) I_{\rho \rho}^{(1)}= & \ln ([1-F] \psi) \ln (F \psi)-\frac{1}{2} \ln ^{2}(F \psi)+\sum_{n=1}^{\infty} \frac{(-1)^{n+1}}{n^{2}}\left(\frac{F}{1-F}\right)^{n}-\frac{\pi^{2}}{6} \\
& -\ln ([1-F] \psi) \ln (1-\rho)-\frac{1}{2} \ln ^{2}(3-\rho)+\ln (3-\rho) \ln (1-\rho) \\
& +\sum_{n=1}^{\infty} \frac{(-1)^{n+1}}{n^{2}}\left(\frac{1-\rho}{3-\rho}\right)^{n} \\
& \quad \text { for } F \leq(1-F), \\
= & \frac{1}{2} \ln ^{2}([1-F] \psi)-\sum_{n=1}^{\infty} \frac{(-1)^{n+1}}{n^{2}}\left(\frac{1-F}{F}\right)^{n}-\ln ([1-F] \psi) \ln (1-\rho) \\
& -\frac{1}{2} \ln ^{2}(3-\rho)+\ln (3-\rho) \ln (1-\rho)+\sum_{n=1}^{\infty} \frac{(-1)^{n+1}}{n^{2}}\left(\frac{1-\rho}{3-\rho}\right)^{n} \\
\quad \text { for } F>(1-F) ; & \\
\left(\frac{3-\rho}{2}\right) I_{\rho \rho}^{(2)}= & \frac{\pi^{2}}{6}+\frac{1}{2} \ln ^{2}\left(\frac{1-\rho}{3-\rho}\right)-\sum_{n=1}^{\infty} \frac{(-1)^{n+1}}{n^{2}}\left(\frac{1-\rho}{3-\rho}\right)^{n} \cdot
\end{aligned}
$$

Before undoing the differentiations with respect to $\rho$, we pause to remark on the meaning that can be attached to the derivatives $I_{\rho \rho}$ and $I_{\rho}$. The second derivative $I_{\rho \rho}$ can be interpreted physically [after multiplication by $N_{\mathrm{ch}} U_{2}(t / \delta)^{4}$ ] as reflecting a change in the effective differential charging energy $U_{\text {eff }}=2\left[\partial^{2} E_{g}^{(0)}(\rho) / \partial \rho^{2}\right]_{\rho=0}$, where $E_{g}^{(0)}(\rho)$ is the ground-state energy as a function of $\rho$ for a given value of the dimensionless channel conductance $g$. (One might choose to speak of an effective differential capacitance $C_{\text {eff }}=e^{2} / 2 U_{\text {eff. }}$ ) Similarly, 610 up to a proportionality factor, the first derivative $I_{\rho}$ can be understood as a tunneling-induced correction to the expectation value of $\hat{n}$.

What is desired here, however, is $I$ itself, $I$ being proportional to Diagram 2's contribution to the fourth-order energy shift [recall Eq. (A99]). Integrating $I_{\rho \rho}$ twice with respect to $\rho$ gives us $I$ up to additive terms that are constant or linear with respect to $\rho$ :

$$
I(\rho, F, \psi)=a_{0}+a_{1} \rho+\int_{0}^{\rho} d x_{1} \int_{0}^{x_{1}} d x_{2} I_{\rho \rho}\left(x_{2}, F, \psi\right) .
$$

As mentioned in Sec. II and at the beginning of this appendix, the unknown terms $\left(a_{0}+a_{1} \rho\right)$ are not relevant to our result. The $a_{1} \rho$ term is negligible due to the existence of the mirror image of Diagram 2, in which the roles of dots 1 and 2 are exchanged. Such a switch of $\hat{n}_{1}$ and $\hat{n}_{2}$ is equivalent -in calculating energies - to taking $\rho \rightarrow-\rho$. Consequently, when the total fourth-order shift is calculated, the $a_{1} \rho$ in Eq. (A17) cancels with the $-a_{1} \rho$ from the mirror image. Likewise, the $a_{0}$ part drops from the final result as we are only concerned with the difference between the energy shifts for arbitrary $\rho$ and $\rho=0$.

The irrelevance of the $a_{0}$ and $a_{1} \rho$ terms tells us that we need only calculate $I$ modulo terms constant or linear with respect to $\rho$. In other words, we need only find an equivalence class

$$
I(\rho, F, \psi) \cong \int_{0}^{\rho} d x_{1} \int_{0}^{x_{1}} d x_{2} I_{\rho \rho}\left(x_{2}, F, \psi\right)
$$


where the congruence symbol indicates equivalence up to additive terms that are constant or linear with respect to $\rho$. We are therefore free to drop any constant or linear terms that crop up on the right side of Eq. (A18).

Confident that we have figured out what we wish to do, we can return to the pedestrian business of doing it. We observe that the $\rho$-dependent sum in Eq. (A16) can be written in a more integrable form:

$$
\begin{aligned}
\sum_{n=1}^{\infty} \frac{(-1)^{n+1}}{n^{2}}\left(\frac{1-\rho}{3-\rho}\right)^{n}= & \sum_{n=1}^{\infty} \frac{(-1)^{n+1}}{n^{2}}\left(\frac{1}{3}\right)^{n}+\ln 2[\ln (1-\rho)-\ln (3-\rho)+\ln 3] \\
& +\int_{0}^{\rho} d x \ln \left(\frac{2-x}{3-x}\right)\left(\frac{-1}{1-x}+\frac{1}{3-x}\right) .
\end{aligned}
$$

Integration of $I_{\rho \rho}^{(1)}$ and $I_{\rho \rho}^{(2)}$ with respect to $\rho$ gives

$$
\begin{aligned}
\frac{1}{2} I_{\rho}^{(1)}= & -\ln (1-\rho)\left[\ln ([1-F] \psi) \ln (F \psi)-\frac{1}{2} \ln ^{2}(F \psi)+\sum_{n=1}^{\infty} \frac{(-1)^{n+1}}{n^{2}}\left(\frac{F}{1-F}\right)^{n}\right. \\
& \left.-\frac{\pi^{2}}{6}+\sum_{n=1}^{\infty} \frac{(-1)^{n+1}}{n^{2}}\left(\frac{1}{3}\right)^{n}+\ln 2 \ln 3\right] \\
& +\frac{1}{2} \ln ^{2}(1-\rho)[\ln ([1-F] \psi)-\ln 2]-\frac{1}{2} \int_{0}^{\rho} d x \frac{\ln ^{2}(3-x)}{1-x} \\
& +\int_{0}^{\rho} d x \frac{\ln (3-x) \ln (1-x)}{1-x}-\ln 2 \int_{0}^{\rho} d x(\rho-x) \frac{\ln (3-x)}{1-x} \\
& +\int_{0}^{\rho} d x_{1} \frac{\rho-x_{1}}{1-x_{1}} \int_{0}^{x_{1}} d x_{2} \ln \left(\frac{2-x_{2}}{3-x_{2}}\right)\left(\frac{-1}{1-x_{2}}+\frac{1}{3-x_{2}}\right) \\
= & -\ln (1-\rho)\left[\frac{1}{2} \ln { }^{2}([1-F] \psi)-\sum_{n=1}^{\infty} \frac{(-1)^{n+1}}{n^{2}}\left(\frac{1-F}{F}\right)^{n}\right. \\
& \left.+\sum_{n=1}^{\infty} \frac{(-1)^{n+1}}{n^{2}}\left(\frac{1}{3}\right)^{n}+\ln 2 \ln 3\right]+\ldots \\
& -\int_{0}^{\rho} d x_{1} \frac{1}{3-x_{1}} \int_{0}^{x_{1}} d x_{2} \ln \left(\frac{2-x_{2}}{3-x_{2}}\right)\left(\frac{-1}{1-x_{2}}+\frac{1}{3-x_{2}}\right) \\
\frac{1}{2} I_{\rho}^{(2)}= & {[-\ln (3-\rho)+\ln 3]\left[\frac{\pi^{2}}{6}-\sum_{n=1}^{\infty} \frac{(-1)^{n+1}}{n^{2}}\left(\frac{1}{3}\right)^{n}-\ln 2 \ln 3\right] } \\
& -\frac{1}{6}\left[\ln ^{3}(3-\rho)-\ln ^{3} 3\right]-\frac{1}{2} \ln 2\left[\ln ^{2}(3-\rho)-\ln { }^{2} 3\right] \\
& +\frac{1}{2} \int_{0}^{\rho} d x \frac{\ln ^{2}(1-x)}{3-x}-\int_{0}^{\rho} d x \frac{\ln ^{2}(3-x) \ln (1-x)}{3-x} \\
& -\ln 2 \int_{0}^{\rho} d x \frac{\ln (1-x)}{3-x} \\
& \\
& \\
& \\
& \\
&
\end{aligned}
$$

The ellipsis in the second equation for $I_{\rho}^{(1)}$ indicates that the remainder of $I_{\rho}^{(1)}$ for the system above half-filling is the same as the corresponding remainder for the system below or at half-filling.

In deriving Eq. A20, we eliminated a number of integrals over $x_{i}$ 's by using an identitye that is easily derived for double integrals:

$$
\int_{0}^{\rho} d x_{1} \int_{0}^{x_{1}} d x_{2} f\left(x_{2}\right)=\int_{0}^{\rho} d x(\rho-x) f(x) .
$$

Nonetheless, in the final terms of $I_{\rho}^{(1)}$ and $I_{\rho}^{(2)}$, double integrals remain. These can be reduced to single-integral form with a little extra work. Defining $L^{(m)}$ to be the last term of $(1 / 2) I_{\rho}^{(m)}$, we discover that 


$$
\begin{aligned}
& L^{(1)}=\int_{0}^{\rho} d x_{2} \ln \left(\frac{2-x_{2}}{3-x_{2}}\right)\left(\frac{-1}{1-x_{2}}+\frac{1}{3-x_{2}}\right) \int_{x_{2}}^{\rho} d x_{1}\left(1-\frac{1-\rho}{1-x_{1}}\right) \\
& =\int_{0}^{\rho} d x \ln \left(\frac{2-x}{3-x}\right)\left(\frac{-1}{1-x}+\frac{1}{3-x}\right) \\
& \times\left[(\rho-x)+(1-\rho) \ln \left(\frac{1-\rho}{1-x}\right)\right], \\
& L^{(2)}=-\int_{0}^{\rho} d x \ln \left(\frac{2-x}{3-x}\right)\left(\frac{-1}{1-x}+\frac{1}{3-x}\right) \\
& \times\left[(\rho-x)+(3-\rho) \ln \left(\frac{3-\rho}{3-x}\right)\right] .
\end{aligned}
$$

Now we perform the final integration over $\rho$, deriving $I^{(1)}$ and $I^{(2)}$, where $I \cong I^{(1)}+I^{(2)}$.

$$
\begin{aligned}
& \frac{1}{2} I^{(1)} \cong(1-\rho) \ln (1-\rho)\left[\ln ([1-F] \psi) \ln (F \psi)-\frac{1}{2} \ln ^{2}(F \psi)+\sum_{n=1}^{\infty} \frac{(-1)^{n+1}}{n^{2}}\left(\frac{F}{1-F}\right)^{n}\right. \\
& \left.-\frac{\pi^{2}}{6}+\sum_{n=1}^{\infty} \frac{(-1)^{n+1}}{n^{2}}\left(\frac{1}{3}\right)^{n}+\ln 2 \ln 3+\ln ([1-F] \psi)-\ln 2\right] \\
& -\frac{1}{2}(1-\rho) \ln ^{2}(1-\rho)[\ln ([1-F] \psi)-\ln 2]-\frac{1}{2} \int_{0}^{\rho} d x(\rho-x) \frac{\ln ^{2}(3-x)}{1-x} \\
& +\int_{0}^{\rho} d x(\rho-x) \frac{\ln (3-x) \ln (1-x)}{1-x}-\ln 2 \int_{0}^{\rho} d x(\rho-x) \frac{\ln (3-x)}{1-x} \\
& +(1-\rho) \int_{0}^{\rho} d x \ln \left(\frac{1-\rho}{1-x}\right) \ln \left(\frac{2-x}{3-x}\right)\left(\frac{-1}{1-x}+\frac{1}{3-x}\right) \\
& +\int_{0}^{\rho} d x(\rho-x) \ln \left(\frac{2-x}{3-x}\right)\left(\frac{-1}{1-x}+\frac{1}{3-x}\right) \\
& \text { for } F \leq(1-F) \text {, } \\
& \cong(1-\rho) \ln (1-\rho)\left[\frac{1}{2} \ln ^{2}([1-F] \psi)-\sum_{n=1}^{\infty} \frac{(-1)^{n+1}}{n^{2}}\left(\frac{1-F}{F}\right)^{n}\right. \\
& \left.+\sum_{n=1}^{\infty} \frac{(-1)^{n+1}}{n^{2}}\left(\frac{1}{3}\right)^{n}+\ln 2 \ln 3+\ln ([1-F] \psi)-\ln 2\right]+\ldots \\
& \text { for } F>(1-F) \text {; } \\
& \frac{1}{2} I^{(2)} \cong[(3-\rho) \ln (3-\rho)-3 \ln 3]\left[\frac{\pi^{2}}{6}-\sum_{n=1}^{\infty} \frac{(-1)^{n+1}}{n^{2}}\left(\frac{1}{3}\right)^{n}-\ln 2 \ln 3\right] \\
& +\frac{1}{6}\left[(3-\rho) \ln ^{3}(3-\rho)-3(3-\rho) \ln ^{2}(3-\rho)+6(3-\rho) \ln (3-\rho)\right. \\
& \left.-3 \ln ^{3} 3+9 \ln ^{2} 3-18 \ln 3\right] \\
& +\frac{1}{2} \ln 2\left[(3-\rho) \ln ^{2}(3-\rho)-2(3-\rho) \ln (3-\rho)-3 \ln ^{2} 3+6 \ln 3\right] \\
& +\frac{1}{2} \int_{0}^{\rho} d x(\rho-x) \frac{\ln ^{2}(1-x)}{3-x}-\int_{0}^{\rho} d x(\rho-x) \frac{\ln (3-x) \ln (1-x)}{3-x} \\
& -\ln 2 \int_{0}^{\rho} d x(\rho-x) \frac{\ln (1-x)}{3-x} \\
& -(3-\rho) \int_{0}^{\rho} d x \ln \left(\frac{3-\rho}{3-x}\right) \ln \left(\frac{2-x}{3-x}\right)\left(\frac{-1}{1-x}+\frac{1}{3-x}\right)
\end{aligned}
$$




$$
-\int_{0}^{\rho} d x(\rho-x) \ln \left(\frac{2-x}{3-x}\right)\left(\frac{-1}{1-x}+\frac{1}{3-x}\right) .
$$

We are essentially done. Upon multiplying the sum of $I^{(1)}$ and $I^{(2)}$ by $N_{\mathrm{ch}} U_{2}(t / \delta)^{4}$, we have the relevant contribution from Diagram 2 to the fourth-order energy shift. After so much work, one might wonder whether we have achieved anything more. Providentially, the answer is that, yes, we have. As explained earlier, we have also solved for the contribution from the corresponding mirror-image diagram, which is obtained by replacing $\rho$ with $-\rho$ in Eq. (A23). Perhaps more surprisingly, we have solved for the contributions from another pair of mirror-image terms. A swap of $F$ and $(1-F)$ in Eq. (A9) turns it into the formula for the contribution from Diagram 3 of Fig. 2(b). Thus, exchanging $F$ and $(1-F)$ in Eq. A23) yields the contribution from Diagram 3. A further replacement of $\rho$ with $-\rho$ gives the contribution from Diagram 3's mirror image. The cost of calculating Diagram 2 is high, but at least we benefit from a package deal-4 for the price of 1 .

\section{APPENDIX B: DETAILS OF THE STRONG-COUPLING CALCULATION}

This appendix consists of three parts presenting various calculations described or cited in Sec. III. The first part computes $S_{\mathrm{b}}^{(1)}, S_{\mathrm{b}}^{(2)}, K_{\mathrm{c}}(\tau)$, and $K_{\mathrm{c}}(0)$, thereby producing the results quoted in Eqs. (27) and making explicit the origin of the factor $e^{\gamma}$ that appears in the prefactors of Eqs. (30) and (34). The second part of the appendix provides the derivation of the first strong-coupling energy correction [see Eq. (33)]. The third part derives the second strongcoupling correction [see Eq. (34)].

\section{Calculation of Charge-Channel Averages}

The leap from Eq. (26) to Eq. (27) in Sec. III requires evaluation of the expectation values

$$
\begin{aligned}
& D_{1}=\left\langle\cos \left[\sqrt{\pi} \theta_{\mathrm{c}}(\tau)+\frac{\pi \rho}{2}\right]\right\rangle_{\mathrm{c}}, \\
& D_{2}=\left\langle\cos \left[\sqrt{\pi} \theta_{\mathrm{c}}\left(\tau_{1}\right)+\frac{\pi \rho}{2}\right] \cos \left[\sqrt{\pi} \theta_{\mathrm{c}}\left(\tau_{2}\right)+\frac{\pi \rho}{2}\right]\right\rangle_{\mathrm{c}} .
\end{aligned}
$$

[Recall that time-ordering is implicit in the path-integral definition of $\langle\hat{A}\rangle_{\mathrm{c}}$ in Eq. (24).] The cosines and products of cosines can be written as linear combinations of terms of the form $e^{\hat{Z}}$, where $\hat{Z}$ is linear in the charge displacement operators $\theta_{\mathrm{c}}(\tau)$ and the charge displacement operators are themselves linear in boson creation and annihilation operators (see Ref. 21). Therefore, one fan apply a standard relation for the expectation value of the exponential of a linear combination of boson operators 22

$$
\left\langle e^{\hat{Z}}\right\rangle=e^{\frac{1}{2}\left\langle\hat{Z}^{2}\right\rangle}
$$

which can easily be shown to hold for our charge-integration brackets with implicit time-ordering.

Using Eq. (B2), we discover that

$$
\begin{aligned}
& D_{1}=e^{-\frac{\pi}{2} K_{\mathrm{c}}(0)} \cos \left(\frac{\pi \rho}{2}\right), \\
& D_{2}=\frac{1}{2} e^{-\pi\left[K_{\mathrm{c}}(0)+K_{\mathrm{c}}\left(\tau_{1}-\tau_{2}\right)\right]} \cos (\pi \rho)+\frac{1}{2} e^{-\pi\left[K_{\mathrm{c}}(0)-K_{\mathrm{c}}\left(\tau_{1}-\tau_{2}\right)\right]},
\end{aligned}
$$

where $K_{\mathrm{c}}(\tau)$ is the charge-charge correlation function,

$$
K_{\mathrm{c}}(\tau)=\left\langle\theta_{\mathrm{c}}(\tau) \theta_{\mathrm{c}}(0)\right\rangle_{\mathrm{c}}
$$

Eq. (27) of Sec. III follows immediately. We find $S_{\mathrm{b}}^{(1)}$ by replacing $\cos \left[\sqrt{\pi} \theta_{\mathrm{c}}(\tau)+\pi \rho / 2\right]$ in $S_{\mathrm{b}}$ with $D_{1}$. For $S_{\mathrm{b}}^{(2)}$, we recall that

$$
\left\langle\left[S_{\mathrm{b}}-\left\langle S_{\mathrm{b}}\right\rangle_{\mathrm{c}}\right]^{2}\right\rangle_{\mathrm{c}}=\left\langle S_{\mathrm{b}}^{2}\right\rangle_{\mathrm{c}}-\left\langle S_{\mathrm{b}}\right\rangle_{\mathrm{c}}^{2}
$$

and apply the formulas for $D_{1}$ and $D_{2}$ accordingly. 
To get the formula for $K_{\mathrm{c}}(\tau)$ [Eq. (28)], we must labor a bit more. Because the unperturbed action $S_{0}^{(\mathrm{c})}$ is quadratic in charge displacement operators $\tilde{\theta}_{\mathrm{c}}\left(\omega_{m}\right), S_{0}^{(\mathrm{c})}$ fits exactly the form for the canonical action of a real scalar field. 36 Consequently,

$$
\left\langle\tilde{\theta}_{\mathrm{c}}\left(\omega_{m}\right) \tilde{\theta}_{\mathrm{c}}\left(-\omega_{n}\right)\right\rangle_{\mathrm{c}}=\frac{\beta}{\left|\omega_{m}\right|+\frac{2 U_{2}}{\pi}} \delta_{\omega_{m}, \omega_{n}} .
$$

From this identity and the relation between $\theta_{\mathrm{c}}(\tau)$ and its Fourier transform [recall Eq. (18)], we construct a summation formula for $K_{\mathrm{c}}(\tau)$ :

$$
K_{\mathrm{c}}(\tau)=\frac{1}{\beta} \sum_{\omega_{m}} \frac{e^{-i \omega_{m} \tau}}{\left|\omega_{m}\right|+\frac{2 U_{2}}{\pi}} .
$$

In the zero-temperature $(\beta \rightarrow \infty)$ limit, we may safely transform this sum into an integral. Before doing so, however, we should note that, unless the $\omega_{m}$ possess an ultraviolet cut-off, $K_{\mathrm{c}}(0)$ diverges logarithmically. The standard means of imposing such a cut-off in Luttinger liquid theory 22 is to insert a factor of $e^{-\left|\omega_{m}\right| / W}$ on the right side of Eq. (18). This insertion generates a factor of $e^{-2\left|\omega_{m}\right| / W}$ in Eq. (B6), yielding

$$
K_{\mathrm{c}}(\tau)=\int_{-\infty}^{\infty} \frac{d \omega}{2 \pi} \frac{e^{-i \omega \tau} e^{-\frac{2|\omega|}{W}}}{|\omega|+\frac{2 U_{2}}{\pi}},
$$

which is equivalent to Eq. (28) in Sec. III.

The way is clear for evaluation of the same-time corrrelation function $K_{\mathrm{c}}(0)$. After setting $\tau=0$ in Eq. (B7), we integrate by parts and convert to the dimensionless integration variable $x=2 \omega / W$. The result is that

$$
\begin{aligned}
K_{\mathrm{c}}(0) & =-\frac{1}{\pi} \ln \left(\frac{4 U_{2}}{\pi W}\right)+\frac{1}{\pi} \int_{0}^{\infty} d x e^{-x} \ln \left(x+\frac{4 U_{2}}{\pi W}\right) \\
& =-\frac{1}{\pi} \ln \left(\frac{4 U_{2}}{\pi W}\right)+\frac{1}{\pi} e^{\left(4 U_{2} / \pi W\right)}\left(\int_{0}^{\infty} d x e^{-x} \ln x-\int_{0}^{4 U_{2} / \pi W} d x e^{-x} \ln x\right) .
\end{aligned}
$$

The first integral in the parentheses equals the negative of $\gamma$, the Euler-Mascheroni constant.18 The second integral goes to zero as we take the limit $W / U_{2} \rightarrow \infty$. In this limit, the exponential factor multiplying the integrals goes to 1. The final result is the following:

$$
K_{\mathrm{c}}(0)=-\frac{1}{\pi} \ln \left(\frac{4 e^{\gamma} U_{2}}{\pi W}\right) .
$$

The derivation of Eq. (B9) shows that the coefficient $e^{\gamma}$ comes from exponentiating a secondary part of $\left\langle\theta_{\mathrm{c}}(0) \theta_{\mathrm{c}}(0)\right\rangle_{\mathrm{T}}$ One might be concerned that Luttinger liquid theory does not faithfully capture such subsidiary dependences. 37 However, Sec. IV presents evidence that these coefficients are general and independent of the high-energy band structure.

\section{The First Strong-Coupling Correction}

As stated in Sec. III, in the limit of strong coupling $(g \rightarrow 1)$, the first correction [see Eq. (33)] to the open-channel $(g=0)$ ground-state energy is obtained by diagonalizing the Hamiltonian $H_{\text {New }}$ [see Eq. (29)]. This diagonalization can be accomplished through another version of the "debosonization" procedure used by Matveev. 9 As we wish to "debosonize" the action $S_{\mathrm{New}}=S_{0}^{(\mathrm{s})}+S_{\mathrm{b}}^{(1)}$ [recall Eqs. 21) and (27)], it is useful to observe that $S_{0}^{(\mathrm{s})}$ corresponds to the Euclidean action for non-interacting fermions on a semi-infinite lattice ending at $x=0.15$ For these fermions, we take $\theta_{\mathrm{S}}(\tau)$ to correspond to the $x=0$ value of the phase field, $\phi_{f}(\tau)=\Phi_{f}(0, \tau)$, rather than the $x=0$ value of the charge displacement field $\theta_{f}(\tau)=\Theta_{f}(0, \tau)$. Making $\theta_{f}(\tau)=0$ the boundary condition at the edge, we find that the properly normalized creation operator for a fermion at $x=0$ is given by

$$
\psi_{f}^{\dagger}(0, \tau)=\sqrt{\frac{W}{4 \pi \hbar v_{F}}} e^{i \sqrt{\pi} \phi_{f}(\tau)},
$$


where, as usual, $W$ is the bandwidth and $v_{F}$ is the Fermi velocity. $15222 \psi_{f}^{\dagger}(0, \tau)$ can be expressed in terms of reciprocalspace creation operators:

$$
\psi_{f}^{\dagger}(0, \tau)=\frac{1}{\sqrt{2 \pi}} \int_{-\Lambda}^{\Lambda} d k f_{k}^{\dagger}
$$

The fermionic energies are cut off in the usual way at $W / 2$, the corresponding wave-vector cut-off being $\Lambda=W / 2 \hbar v_{F}$.

After these machinations, "refermionization" proceeds apace. Since the unperturbed action $S_{0}^{(\mathrm{s})}$ is an action for non-interacting fermions, the unperturbed Hamiltonian $H_{0}^{(\mathrm{s})}$ is simply the sum of the single-particle energies of those fermions. On the other hand, the perturbation $H_{\mathrm{b}}^{(1)}$ that corresponds to $S_{\mathrm{b}}^{(1)}$ is a term linear in fermion creation and annihilation operators. In particular, using Eq. (B9) to determine $e^{-\frac{\pi}{2} K_{\mathrm{c}}(0)}$, we obtain

$$
\begin{aligned}
& H_{0}^{(\mathrm{s})}=\int_{-\Lambda}^{\Lambda} d k \xi_{k} f_{k}^{\dagger} f_{k}, \\
& H_{\mathrm{b}}^{(1)}=\tilde{V} \cos \left(\frac{\pi \rho}{2}\right) \sqrt{\frac{2 e^{\gamma} \hbar v_{F} U_{2}}{\pi^{3}}} \int_{-\Lambda}^{\Lambda} d k\left(f_{k}^{\dagger}+f_{k}\right),
\end{aligned}
$$

Not being quadratic in fermion creation and annihilation operators, the fermionic Hamiltonian we have derived is not yet in an easily diagonalizable form. To make it so, we follow Matveevl in defining a new set of fermion operators such that

$$
f_{k}=\left(d+d^{\dagger}\right) d_{k} .
$$

Plugging this equivalence into Eq. (B12) yields Eq. (30) of Sec. III.

One can now perform the Bogoliubov transformation that produces Eq. (31). To find the correction to the openchannel energy, one notes that $H_{\mathrm{b}}^{(1)}$ of Eq. (30) has an expectation value of zero in the ground state of $H_{0}^{(\mathrm{s})}$, which is the open-channel $(\tilde{V}=0)$ part of $H_{\mathrm{New}}$. Therefore, if the ground state of $H_{0}^{(\mathrm{s})}$ is represented by the ket $|0\rangle$, $\left\langle 0\left|H_{\mathrm{New}}\right| 0\right\rangle=E_{0}$, where $E_{0}$ is the ground-state energy for $H_{0}^{(\mathrm{s})}$. From the diagonalized form of $H_{\mathrm{New}}$ [see Eq. (31)], it is then deduced that the equation for $\left(E_{\mathrm{New}}-E_{0}\right)$ is the following:

$$
\Delta_{\mathrm{str}}^{(1)}(\rho)=-\int_{0}^{\Lambda} d k \xi_{k}\left\langle 0\left|C_{k}^{\dagger} C_{k}+\tilde{C}_{k}^{\dagger} \tilde{C}_{k}\right| 0\right\rangle .
$$

At this point, it is necessary to know the exact equations for $\tilde{C}_{k}$ and $C_{k}$. As found by Matveev $\mathrm{g}$ for $\Gamma=$ $\tilde{V}^{2}\left[8 e^{\gamma} U_{2} / \pi^{2}\right] \cos ^{2}(\pi \rho / 2)$, they are

$$
\begin{aligned}
\tilde{C}_{k}= & \frac{d_{k}+d_{-k}^{\dagger}}{\sqrt{2}}, \\
C_{k}= & \frac{\xi_{k}}{\sqrt{\xi_{k}^{2}+\Gamma^{2}}} \frac{d_{k}-d_{-k}^{\dagger}}{\sqrt{2}}-\sqrt{\frac{\hbar v_{F} \Gamma}{2 \pi\left(\xi_{k}^{2}+\Gamma^{2}\right)}}\left(d+d^{\dagger}\right) \\
& \quad+\frac{\Gamma}{\pi \sqrt{\xi_{k}^{2}+\Gamma^{2}}} \mathcal{P} \int_{-\Lambda}^{\Lambda} \frac{d \xi_{k^{\prime}}}{\xi_{k}-\xi_{k^{\prime}}} \frac{d_{k^{\prime}}-d_{-k^{\prime}}^{\dagger}}{\sqrt{2}},
\end{aligned}
$$

As before, the symbol $\mathcal{P}$ indicates that only the principal value of the integral is computed.

With the explicit equations for $\tilde{C}_{k}$ and $C_{k}$ before us, it is clear that, for $k>0, \tilde{C}_{k}|0\rangle=0$, and

$$
\Delta_{\mathrm{str}}^{(1)}(\rho)=-\int_{0}^{\Lambda} d k \xi_{k}\left\langle 0\left|C_{k}^{\dagger} C_{k}\right| 0\right\rangle .
$$

Concentrating on what remains, we see that, for $k>0$, both the first term of $C_{k}$ and the $k^{\prime}>0$ part of the third term of $C_{k}$ annihilate the $H_{0}^{(\mathrm{s})}$ ground state. Hence,

$$
\begin{aligned}
\left\langle 0\left|C_{k}^{\dagger} C_{k}\right| 0\right\rangle & =\frac{\hbar v_{F} \Gamma}{2 \pi\left(\xi_{k}^{2}+\Gamma^{2}\right)}\left[1+\frac{\Gamma}{\pi \hbar v_{F}} \int_{0}^{\Lambda} \frac{d k^{\prime}}{k+k^{\prime}} \int_{0}^{\Lambda} \frac{d k^{\prime \prime}}{k+k^{\prime \prime}}\left\langle 0\left|\left(d_{-k^{\prime}}^{\dagger}+d_{k^{\prime}}\right)\left(d_{-k^{\prime \prime}}+d_{k^{\prime \prime}}^{\dagger}\right)\right| 0\right\rangle\right] \\
& =\frac{\hbar v_{F} \Gamma}{2 \pi\left(\xi_{k}^{2}+\Gamma^{2}\right)}+\frac{\Gamma^{2}}{\pi^{2}\left(\xi_{k}^{2}+\Gamma^{2}\right)}\left(\frac{1}{k}-\frac{1}{k+\Lambda}\right) .
\end{aligned}
$$


Plugging into Eq. (B16), we find that

$$
\begin{aligned}
\Delta_{\mathrm{str}}^{(1)}(\rho)= & -\frac{\Gamma}{2 \pi} \int_{0}^{W / 2} \frac{\xi_{k} d \xi_{k}}{\xi_{k}^{2}+\Gamma^{2}}-\frac{\Gamma^{2}}{\pi^{2}} \int_{0}^{W / 2} \frac{d \xi_{k}}{\xi_{k}^{2}+\Gamma^{2}} \\
& +\frac{\Gamma^{2}}{\pi^{2}} \int_{0}^{W / 2} \frac{\xi_{k} d \xi_{k}}{\left(\xi_{k}+\frac{W}{2}\right)\left(\xi_{k}^{2}+\Gamma^{2}\right)} \\
= & -\frac{\Gamma}{4 \pi} \ln \left(\frac{W^{2}}{4 \Gamma^{2}}+1\right)-\frac{\Gamma}{2 \pi} \\
= & -\frac{\Gamma}{2 \pi}\left[\ln \left(\frac{W}{2 \Gamma}\right)+1\right] .
\end{aligned}
$$

Here we have dropped terms that vanish as $W / U_{2} \rightarrow \infty$. Eq. (33) is obtained by applying the identity $\Gamma=$ $\tilde{V}^{2}\left[8 e^{\gamma} U_{2} / \pi^{2}\right] \cos ^{2}(\pi \rho / 2)$.

\section{The Second Strong-Coupling Correction}

The second correction term in the strong-coupling limit [see Eq. (34)] is derived by treating $S_{\mathrm{b}}^{(2)}$ [see Eq. (27)] as a perturbation to the system described by $H_{\mathrm{New}}$ of Eq. (29). Using the standard formula for the grand-canonical potential in the finite-temperature path-integral approach 38

$$
\Omega-\Omega_{0}=-\frac{1}{\beta} \sum \text { (All connected graphs), }
$$

we see that the lowest-order correction to the ground state energy of $H_{\mathrm{New}}$ is given by

$$
\Delta_{\mathrm{str}}^{(2)}(\rho)=\lim _{\beta \rightarrow \infty} \frac{1}{\beta}\left\langle\mathrm{New}\left|S_{\mathrm{b}}^{(2)}\right| \mathrm{New}\right\rangle,
$$

where $|\mathrm{New}\rangle$ is the ground-state ket for $H_{\mathrm{New}}$. The minus sign in Eq. (B19) has been canceled by the minus sign that arises from the fact that this leading term from $S_{\mathrm{b}}^{(2)}$ corresponds to a first-order graph and therefore carries a factor of -1.38

Recalling Eq. (27) and observing that the parts of $S_{\mathrm{b}}^{(2)}$ that are independent of $\rho$ are irrelevant to calculation of the fractional peak splitting $f$, our immediate task is to evaluate the quantity

$$
X\left(\tau_{1}, \tau_{2}\right)=\left(\frac{\tilde{V} W}{\pi}\right)^{2} e^{-\pi K_{\mathrm{c}}(0)} \cos ^{2}\left(\frac{\pi \rho}{2}\right)\left\langle\mathrm{New}\left|\cos \left[\sqrt{\pi} \theta_{\mathrm{s}}\left(\tau_{1}\right)\right] \cos \left[\sqrt{\pi} \theta_{\mathrm{s}}\left(\tau_{1}\right)\right]\right| \mathrm{New}\right\rangle .
$$

Under "debosonization" (see Part 2 of this appendix), this becomes

$$
\begin{gathered}
X\left(\tau_{1}, \tau_{2}\right)=\lambda^{2} \int_{-\Lambda}^{\Lambda} d k_{1} \int_{-\Lambda}^{\Lambda} d k_{2}\langle\mathrm{New}|\left[d_{k_{1}}^{\dagger}\left(d+d^{\dagger}\right)+\left(d+d^{\dagger}\right) d_{k_{1}}\right]_{\tau_{1}} \\
\times\left[d_{k_{2}}^{\dagger}\left(d+d^{\dagger}\right)+\left(d+d^{\dagger}\right) d_{k_{2}}\right]_{\tau_{2}}|\mathrm{New}\rangle,
\end{gathered}
$$

where the bracket subscripts indicate that the enclosed operators are evaluated at imaginary times $\tau_{1}$ and $\tau_{2}$, respectively, and we have used

$$
\lambda=\tilde{V} \cos (\pi \rho / 2) \sqrt{2 e^{\gamma} \hbar v_{F} U_{2} / \pi^{3}} .
$$

We are now within hailing distance of Eq. (34). Using the truncated equations for $C_{k}$ and $\tilde{C}_{k}$ [recall Eq. (32)], we express the $d_{k}$ 's in terms of these operators. The sub-leading terms in this transformation are negligible as, in the end result, they take us beyond second order in $\tilde{V}$. Similarly, the time-dependence of the operator sum $\left(d+d^{\dagger}\right)$ is sub-leading as $\left(d+d^{\dagger}\right)$ first appears in the expansion of the diagonalizing operators at order $\tilde{V}$. Accordingly, $\left(d+d^{\dagger}\right)$ commutes with $H_{\mathrm{New}}$ to zeroth order and can be considered time-independent. In contrast, from Eq. (31), we know that $C_{k}(\tau)=C_{k} e^{-\xi_{k} \tau}$ and $C_{k}^{\dagger}(\tau)=C_{k}^{\dagger} e^{\xi_{k} \tau}$. Application of these insights to Eq. (B22) gives 


$$
\begin{aligned}
X\left(\tau_{1}, \tau_{2}\right) & =2 \lambda^{2} \int_{0}^{\Lambda} d k_{1} \int_{0}^{\Lambda} d k_{2}\left\langle\text { New }\left|C_{k_{1}}\left(\tau_{1}\right) C_{k_{2}}^{\dagger}\left(\tau_{2}\right)\right| \text { New }\right\rangle \\
& =\frac{2 \lambda^{2}}{\hbar v_{F}} \int_{0}^{W / 2} d \xi e^{-\left(\tau_{1}-\tau_{2}\right) \xi} \\
& =\frac{2 \lambda^{2}}{\hbar v_{F}} \frac{1-e^{-\left(\tau_{1}-\tau_{2}\right) W / 2}}{\tau_{1}-\tau_{2}} .
\end{aligned}
$$

We now return to Eqs. (27) and (B20). Switching to dimensionless variables $x_{i}=\tau_{i} W / 2$ and substituting for $\lambda$, we obtain

$$
\begin{aligned}
\Delta_{\mathrm{str}}^{(2)}(\rho)=\tilde{V}^{2} \cos ^{2}\left(\frac{\pi \rho}{2}\right) \frac{8 e^{\gamma} U_{2}}{\pi^{3} \beta W} & \int_{0}^{\beta W / 2} d x_{1} \int_{0}^{x_{1}} d x_{2} \\
& \times\left(1-e^{-\pi K_{\mathrm{c}}\left[2\left(x_{1}-x_{2}\right) / W\right]}\right) \frac{1-e^{-\left(x_{1}-x_{2}\right)}}{x_{1}-x_{2}} .
\end{aligned}
$$

We eliminate one of the integrations by expressing the integrand in terms of $x=\left(x_{1}-x_{2}\right)$ and observing that in the double-integral the density of states for a given value of $x$ is $(\beta W / 2-x)$ :

$$
\Delta_{\mathrm{str}}^{(2)}(\rho)=\tilde{V}^{2} \cos ^{2}\left(\frac{\pi \rho}{2}\right) \frac{8 e^{\gamma} U_{2}}{\pi^{3} \beta W} \int_{0}^{\beta W / 2} d x\left(\frac{\beta W}{2}-x\right)\left[1-e^{-\pi K_{\mathrm{c}}(2 x / W)}\right] \frac{1-e^{-x}}{x} .
$$

Transformation of Eq. (B26) into Eq. (34) follows recognition of the fact that, for $x$ on the order of $\beta W / 2$, the integrand is effectively zero. This is known from the identity

$$
K_{\mathrm{c}}\left(\frac{2 x}{W}\right)=-\frac{1}{\pi} \operatorname{Re}\left\{e^{\left(4 U_{2} / \pi W\right)(1+i x)} \operatorname{Ei}\left[-\left(4 U_{2} / \pi W\right)(1+i x)\right]\right\},
$$

where $\operatorname{Ei}[-z]$ is the first exponential integral function. 39 For $z \gg 1, \operatorname{Ei}[-z]$ goes as $e^{-z} / z$. Therefore, the integrand goes to zero as $1 / x^{2}$ for $x>\pi W / 4 U_{2}$, and the region $x \gg W / U_{2}$ makes a comparatively negligible contribution to the integral. This conclusion corroborates the statement made in Sec. III that the factor $\left[1-e^{-\pi K_{\mathrm{c}}(2 x / W)}\right]$ furnishes an ultraviolet cut-off on the order of $\psi=W / U_{2}$. Since we calculate in the limit $\beta \rightarrow \infty$, we know that $\beta W / 2 \gg W / U_{2}$ and, hence, that the integrand is effectively zero for $x$ on the order of $\beta W / 2$. We can approximate the weight function $(\beta W / 2-x)$ by $(\beta W / 2)$. The result is Eq. (34). 
${ }^{1}$ For an introduction to "single-electronics," see M. A. Kastner, Rev. Mod. Phys. 64, 849 (1992); D. V. Averin and K. K. Likharev, in Mesoscopic Phenomena in Solids, edited by B. L. Altshuler, P. A. Lee, and R. A. Webb (North Holland, Amsterdam, 1991); and several articles in Single Charge Tunneling, Vol. 294 of NATO Advanced Study Institute Series B Physics, edited by H. Grabert and M. H. Devoret (Plenum, New York, 1992).

${ }^{2}$ F. R. Waugh, M. J. Berry, D. J. Mar, R. M. Westervelt, K. L. Campman, and A. C. Gossard, Phys. Rev. Lett. 75, 705 (1995).

${ }^{3}$ F. R. Waugh, M. J. Berry, C. H. Crouch, C. Livermore, D. J. Mar, R. M. Westervelt, K. L. Campman, and A. C. Gossard, Phys. Rev. B 53, 1413 (1996); F. R. Waugh, Ph.D. thesis, Harvard University, 1994.

${ }^{4}$ J. M. Golden and B. I. Halperin, Phys. Rev. B 53, 3893 (1996).

${ }^{5}$ K. A. Matveev, L. I. Glazman, and H. U. Baranger, Phys. Rev. B 53, 1034 (1996).

${ }^{6}$ K. A. Matveev, L. I. Glazman, and H. U. Baranger, preprint (12/11/95, cond-mat/9512082.

${ }^{7}$ D. S. Golubev and A. D. Zaikin, Phys. Rev. B 50, 8736 (1994).

8 Hermann Grabert, Phys. Rev. B 50, 17364 (1994); Physica B 194-196, 1011-1012 (1994).

${ }^{9}$ K. A. Matveev, Phys. Rev. B 51, 1743 (1995).

${ }^{10}$ L. W. Molenkamp, Karsten Flensberg, and M. Kemerink, Phys. Rev. Lett. 75, 4282 (1995).

${ }^{11}$ Karsten Flensberg, Physica B 203, 432 (1994); Phys. Rev. B 48, 11156 (1993).

${ }^{12}$ G. Falci, J. Heins, Gerd Schön, and Gergely T. Zimanyi, Physica B 203, 409 (1994); G. Falci, Gerd Schön, and Gergely T. Zimanyi, Phys. Rev. Lett. 74, 3257 (1995).

13 S. V. Panyukov and A. D. Zaikin, Phys. Rev. Lett. 67, 3168 (1991); Physics Letters A 183, 115 (1993).

${ }^{14}$ H. O. Frota and Karsten Flensberg, Phys. Rev. B 46, 15207 (1992).

${ }^{15}$ C. L. Kane and M. P. A. Fisher, Phys. Rev. Lett. 68, 1220 (1992); Phys. Rev. B 46, 7268 (1992); ibid. 46, 15233 (1992).

${ }^{16}$ Gordon Baym, Lectures on Quantum Mechanics (AddisonWesley, Redwood City, 1973), $113 \mathrm{ff}$.

${ }^{17}$ M. P. A. Fisher and Wilhelm Zwerger, Phys. Rev. B 32, 6190 (1985).

${ }^{18}$ Murray R. Speigel, Mathematical Handbook of Formulas and Tables, Schaum's Outline Series (McGraw-Hill, New York, 1992).

${ }^{19}$ L. I. Glazman and K. A. Matveev, Zh. Éksp. Teor. Fiz. 98, 1834 (1990) [Sov. Phys. JETP 71, 1031 (1990)]; K. A. Matveev, ibid. 99, 1598 (1991) [72, 892 (1991)]
${ }^{20}$ W. H. Press, S. A. Teukolsky, W. T. Vetterling, and B. P. Flannery, Numerical Recipes in C: The Art of Scientific Computing, 2nd ed. (Cambridge University Press, Cambridge, 1992), 161.

${ }^{21}$ F. D. M. Haldane, Phys. Rev. Lett. 47, 1840 (1981); J. Phys. C 14, 2585 (1981).

${ }^{22}$ V. J. Emery, "Theory of the One-Dimensional Electron Gas," in Highly Conducting One-Dimensional Solids, edited by J. T. Devreese, R. P. Evrard, and V. E. van Doren (Plenum, New York, 1979), 247-303.

${ }^{23}$ I. M. Ruzin, V. Chandrasekhar, E. I. Levin, and L. I. Glazman, Phys. Rev. B 45, 13469 (1992); L. I. Glazman and V. Chandrasekhar, Europhys. Lett. 19, 623 (1992).

${ }^{24}$ C.J.B. Ford, P.J. Simpson, M. Pepper, D. Kern, J.E.F. Frost, D.A. Ritchie, and G.A.C. Jones, Nanostructured Materials 3, 283 (1993).

${ }^{25}$ C. A. Stafford and S. Das Sarma, Phys. Rev. Lett. 72, 3590 (1994).

${ }^{26}$ C. A. Stafford and S. Das Sarma, preprint (5/12/95, condmat/9505058).

${ }^{27}$ M. Kemerink and L.W. Molenkamp, Appl. Phys. Lett. 65, 1012 (1994).

${ }^{28}$ N. Tsukada, M. Gotoda, M. Nunoshita, Phys. Rev. B 50, 5764 (1994).

29 T. Sakamoto, S.W. Hwang, F. Nihey, Y. Nakamura, and K. Nakamura, Jpn. J. Appl. Phys. 33, 4876 (1994); Superlattices and Microstructures 16, 291 (1994).

${ }^{30}$ G. Klimeck, Guanlong Chen, and S. Datta, Phys. Rev. B 50, 2316 (1994); Guanlong Chen, G. Klimeck, S. Datta, Guanha Chen, and W. A. Goddard III, ibid. 50, 8035 (1994).

${ }^{31}$ F. Hofmann, T. Heinzel, D.A. Wharam, J.P. Kotthaus, G. Böhm, W. Klein, G. Tränkle, and G. Weimann, Phys. Rev. B 51, 13872 (1995).

32 R.J. Haug, R.H. Blick, and T. Schmidt, Physica B 212, 207 (1995).

${ }^{33}$ N.C. van der Vaart, S.F. Godijn, Y.V. Nazarov, C.J.P.M. Harmans, J.E. Mooij, L.W. Molenkamp, C.T. Foxon, Phys. Rev. Lett. 74, 4702 (1995).

${ }^{34}$ C. H. Crouch, C. Livermore, F. R. Waugh, R. M. Westervelt, K. L. Campman, and A. C. Gossard, Proceedings of Electronic Properties of Two-Dimensional Systems XI [Surf. Sci. (to be published)].

35 P. Pals and A. MacKinnon, preprint (12/31/96, condmat/9601155); preprint (12/31/96, cond-mat/9601156.

${ }^{36}$ D. J. Amit, Field Theory, the Renormalization Group, and Critical Phenomena, 2nd ed. (World Scientific, Singapore, 1984).

37 J. Solyom, Adv. Phys. 28, 201 (1979).

38 J. W. Negele and H. Orland, Quantum Many-Particle Systems (Addison-Wesley, Redwood City, 1988).

${ }^{39}$ I. S. Gradshteyn and I. M. Ryzhik, Table of Integrals, Series, and Products, 5th ed., edited by Alan Jeffrey (Academic, Boston, 1994), 933ff. 